\title{
Shear Alfvén fluctuation spectrum in Divertor Tokamak Test facility plasmas
}

\author{
T. Wang, ${ }^{1}$ Z. Qiu, ${ }^{1}$ F. Zonca, ${ }^{2,1,}$ a) S. Briguglio, ${ }^{2}$ G. Fogaccia, ${ }^{2}$ G. Vlad, ${ }^{2}$ and X. Wang ${ }^{3}$ \\ ${ }^{1)}$ Institute for Fusion Theory and Simulation and Department of Physics, Zhejiang University, Hangzhou 310027, \\ China \\ ${ }^{2)}$ ENEA, Fusion and Nuclear Safety Department, C. R. Frascati, Via E. Fermi 45, 00044 Frascati (Roma), \\ Italy \\ ${ }^{3)}$ Max-Planck-Institut für Plasmaphysik, Boltzmannstr. 2, 85748 Garching, Germany.
}

The Divertor Tokamak Test (DTT) facility is proposed for studying power exhaust solutions as well as integrated physics and technology aspects for the demonstration power plant (DEMO). To illuminate the richness of new novel plasma physics that can be explored in this device, linear stability properties and shear Alfvén fluctuation spectra of a typical DTT reference scenario are investigated by self-consistent hybrid magnetohydrodynamic-gyrokinetic simulations. The DTT core plasma can be divided into two regions, characterized by reverse shear Alfvén eigenmode in the central core, and by toroidal Alfvén eigenmode in the outer core region. The non-perturabtive effect of energetic particles (EPs), the wave-EP resonance condition as well as power transfer are analyzed in great detail, demonstrating the peculiar role played by EPs in multi-scale dynamics. The most unstable mode number of dominant Alfvénic fluctuations are shown to be of the order of 10, consistent with the typical orbit widths of the EPs normalized to the plasma minor radius and the DTT target design.

\section{INTRODUCTION}

The Divertor Tokamak Test (DTT) facility ${ }^{1-4}$ is a proposal for a next generation tokamak device, which mainly aims at investigating viable and/or advanced power exhaust solutions for the demonstration power plant (DEMO $)^{5}$, thereby filling gaps between present day devices, the International Thermonuclear Experimental Reactor (ITER) $)^{6,7}$ and DEMO. Although DTT is designed as a relatively compact machine (major radius $R_{0}=2.15 \mathrm{~m}$ ) focused on divertor and power exhaust issues, it is capable to reproduce edge as well as core plasma conditions that are relevant for burning plasmas studies $^{4}$. This is, in fact, the prerequisite for studying integrated physics and technology aspects compatible with DEMO operation. Ultimately, this integrated physics and technology approach is reflected in terms of a set of dimensionless parameters, which dictate the key physics and are a crucial element of the present work ${ }^{4}$. In particular, in DTT plasmas, there exists substantial integrated core and edge plasma physics for the reasons discussed in Refs. 1 and 8, which are assumed here as a starting point and whose motivation is outside the intended scope the present work. Thus, among various important issues, key physics aspects to investigate and understand for illuminating the complex plasma behavior ${ }^{9}$ are the properties of the Alfvénic fluctuation spectra in DTT and the related dynamics of energetic particles (EPs) produced by fusion reaction and/or auxiliary heating methods. These supra-thermal plasma components are characterized by typical dimensionless orbit widths (characteristic physical particle Larmor radius as well as magnetic drift orbit size normalized to the machine size) similar to those expected in burning fusion plasmas; and generally smaller

a)Electronic mail: fulvio.zonca@enea.it than in present day tokamaks. Also the ratio of characteristic supra-thermal ion speed to the Alfvén speed in DTT is similar to that expected in ITER and DEMO in order to preserve the strength of EP drive of Alfvénic fluctuations via wave-EP resonant interactions. These key physics elements will result into integrated physics behaviors of DTT plasmas similar to those expected in the proposed next generation tokamaks, for example, in ITER and Chinese Fusion Engineering Test Reactor $(\text { CFETR })^{10,11}$.

In the past several decades, the collective excitations of shear Alfvén wave (SAW) by EPs have been vastly studied in the literature. Recent reviews discuss the general theoretical framework ${ }^{9}$, experimental evidence and their interpretation and modeling ${ }^{12,13}$, as well as comparisons of numerical simulation results with observations ${ }^{14,15}$. These Alfvénic fluctuations exist either as discretized Alfvén eigenmodes (AEs) ${ }^{16}$ or energetic particle continuum modes $(\mathrm{EPMs})^{17}$, depending on the strength of the EP drive and according to the general classification of Alfvénic fluctuations based on the general fishbone-like dispersion relation $(\text { GFLDR })^{18,19}$. In this paper, we aim at investigating the general features of Alfvénic fluctuation spectra resonantly excited by EPs in DTT. In particular, we focus on the mode structures and linear stability properties of AEs and EPMs, while the nonlinear dynamics and implications for EP transport will be analyzed in a future work. Numerical simulation results, presented here, assume a typical DTT reference scenario and a model "slowing-down" EP distribution function, making use of the hybrid magnetohydrodynamic(MHD)gyrokinetic simulation code (HMGC) ${ }^{20,21}$. HMGC has been extensively applied to study the resonant interactions between SAW and EPs as well as the corresponding nonlinear behaviors and ensuing EP confinement issues $^{22-27}$. HMGC has also been used to investigate plasma scenarios of practical interest, such as ITER ${ }^{28}$, Japan Atomic Energy Research Institute Tokamak-60 
Upgrade (JT-60U) ${ }^{29-31}$, Doublet III-D (DIII-D) ${ }^{32,33}$ and Fusion Advanced Studies Torus (FAST) ${ }^{8,34,35}$. As noted above, the main focus of DTT is "power exhaust" and "plasma wall interactions". Nonetheless, based on the same spirit of studying ITER and DEMO relevant burning plasma physics in plasmas with similar dimensionless parameters, it seems natural to adopt a FAST-reference ${ }^{8}$ equilibrium in our simulations along with ITER-like EP pressure profiles. In fact, core plasma profiles and dimensionless parameters of FAST and DTT are very similar ${ }^{1-4,8}$. Menwhile, FAST additional heating was designed to achieve ITER-like EP pressure profiles in a tokamak with similar size and shape to DTT ${ }^{8}$. Thus, although DTT heating system has not been finalized yet, it is meaningful to address the physics that can be investigated in DTT assuming in the present work the same ITER-like EP pressure profiles ${ }^{8}$. We extend our numerical studies to higher toroidal mode numbers than those observed and expected in present days machines, consistent with the DTT target design. In particular, we focus on linear stability, AE spectrum, and waveparticle resonance condition as well as power exchange. In the present work, we illuminate the interplay among shear Alfvén continuum, resonance condition, and most unstable (high) mode numbers. Energetic particles are active players on the length scale of EP magnetic drift orbit widths; i.e. meso-scales intermediate between the macro-scales of plasma equilibrium profiles and plasma turbulence micro-scales ${ }^{9}$. Not only they resonantly excite plasma instabilities but can cause meso-scale modulations in the thermal plasma profiles, thereby playing a crucial role in turbulent transport regulation ${ }^{9}$. Meanwhile, because of fluctuation induced EP transport on meso-scales even longer than the EP characteristic orbit widths, EPs eventually influence fusion reactivity profiles $^{9}$. Thus, our linear stability analysis, emphasizing the importance of high toroidal mode number fluctuations, suggests the crucial role played by meso-spatial and -temporal scales in the nonlinear regime ${ }^{9}$ as will be discussed in a future publication. Thus, our analysis concentrates on some aspects of core physics, but suggests the substantial integration of core and edge plasma physics in DTT, due to the unique role played by fast particles as mediators of cross-scale couplings and by the meso-scales in the transport processes ${ }^{36}$, eventually affecting power exhaust.

This paper is organized as follows. Section II describes the simulation model and main parameters. In Section III, the linear stability property and shear Alfvén fluctuation spectrum of the proposed DTT reference scenario are analyzed, focusing on both the macroscopic mode dynamics and microscopic wave-particle resonant interactions. Finally, summary and discussion are presented in Section IV.

\section{SIMULATION MODEL AND EQUILIBRIUM PARAMETERS}

HMGC is a hybrid magnetohydrodynamic-gyrokinetic simulation code based on a simplified plasma description ${ }^{37}$, assumed to consist of "a core or thermal plasma component, essentially providing an isotropic Maxwellian background made of electrons (e) and ions (i), and an energetic component (H, stands for "Hot"), which is often anisotropic and non-Maxwellian." 9 The HMGC code adopts a relatively simple geometry; i.e., a high aspect ratio tokamak plasma equilibrium with shifted circular magnetic surfaces. In fact, in its simplest form, HMGC describes the core plasmas by a set of $O\left(\epsilon^{3}\right)$-reduced MHD equations ${ }^{38}$, with $\epsilon \equiv a / R_{0} \ll 1$ the inverse aspect ratio and $a$ the tokamak minor radius. Furthermore, the core plasma equilibrium is typically assumed to be cold, with $T_{e}=T_{i}=0$. This is the case we consider in this work, although an extended version of the HMGC code exist, which can treat thermal electrons as massless fluid and thermal ions as a drift-kinetic species $^{39}$. Meanwhile, EPs are treated by a nonlinear drift-kinetic description ${ }^{20,21}$, which is used to compute the corresponding pressure tensor that, in turn, affects the overall plasma response via the force balance in the so-called pressure-coupling formulation ${ }^{40}$. The physics basis for the hybrid MHD-gyrokinetic description and its relevance for burning fusion plasmas is reviewed and discussed in Ref. 9.

The main aim of the present work is to illustrate the meso-scale features and stability properties of Alfvénic oscillations resonantly excited by fast ions in DTT. Thus, we will limit ourselves to a linear analysis adopting the cold core plasma limit of the HMGC code. Due to the underlying $O\left(\epsilon^{3}\right)$-reduced MHD equations, the reference DTT equilibrium analyzed here is reshaped to a circular one with $\epsilon=0.18$, while the radial profile of safety factor $q$ is kept the same. Note that, although the width of toroidicity induced frequency gaps is somewhat reduced, the main simulation results will not be qualitatively altered as we will demonstrate in the next section. Meanwhile, with this choice we will be able to investigate stability properties of fluctuations with moderate to high toroidal mode numbers, which are of particular interest for the present study of shear Alfvén fluctuations in DTT with normalized EP orbit width much smaller than in present day tokamaks.

As anticipated above, it is natural to adopt a FASTreference equilibrium in our simulations along with ITER-like EP pressure profies. Fig. 1 shows the equilibrium $q$ profile characterized by $q_{\min }=1.02$ at $r_{q_{\min }} / a \simeq 0.332$, and the corresponding magnetic shear, $s \equiv(r / q)(d q / d r)$, profile. Here, $r$ is the minor radial coordinate. This equilibrium shows that the plasma can be divided into two regions, i.e., a central core region with nearly flat $q$ profile slightly in excess of $q=1$, and an outer core region with larger $q$ and finite shear. This schematic subdivision can be further demonstrated by 


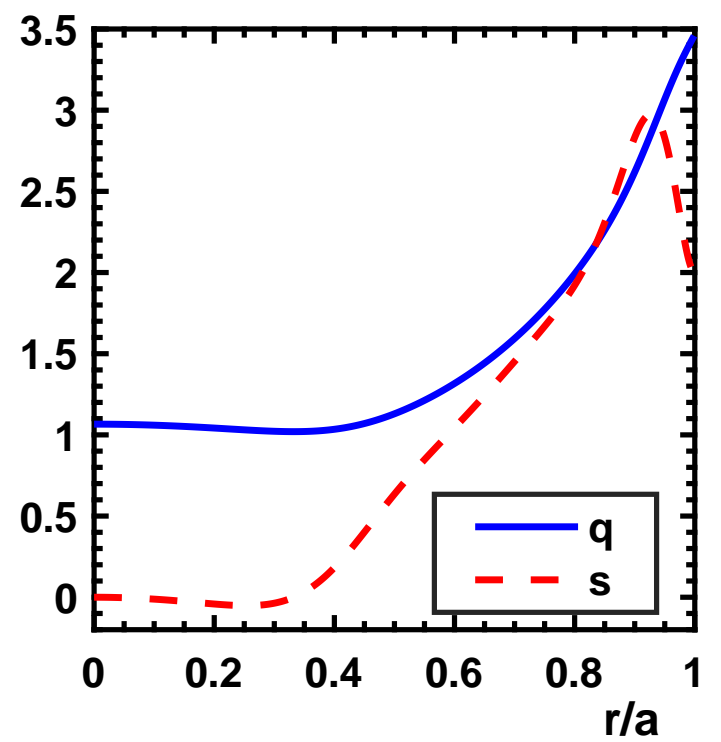

FIG. 1. Radial profiles of safety factor $q$ (blue curve) and magnetic shear $s$ (dashed red curve) adopted in the simulations.

the features of the SAW continuous spectrum. As an example, the SAW continua for $n=2$ and $n=10$ are shown in Fig. 2, along with the radial profile of bulk ion density $n_{i}$, which is assumed to be deuterium. The structures of the SAW continua also demonstrate the existence of low frequency gaps induced by reverse shear in the central core region, and of higher frequency toroidicity induced frequency gaps in the outer core region.

EP dynamics are treated non-perturbatively in HMGC and, as discussed above, they are described by solving nonlinear Vlasov equation in the drift-kinetic limit via particle-in-cell methods. Finite Larmor radius (FLR) effects are neglected, but finite drift orbit width (FOW) effects are consistently retained ${ }^{9,20,21}$. In our simulations, EPs are assumed to be characterized by an isotropic slowing down initial distribution function with EP birth energy $E_{0}$ given by fusion energy of alpha particles and critical energy $E_{c}$ given by Stix expression ${ }^{41}$. The initial $\mathrm{EP}$ distribution function is in the form:

$$
F_{H} \propto \frac{n_{H}(\psi)}{E_{c}^{3 / 2}} \frac{1}{\left[\left(E / E_{c}\right)^{3 / 2}+1\right] \ln \left[\left(E_{0} / E_{c}\right)^{3 / 2}+1\right]} .
$$

Here, $n_{H}(\psi)$ is the radial density profile, $E=m_{H} U^{2} / 2+$ $M \Omega_{H}$ is the particle energy, where $m_{H}$ is the alpha particle mass, $U$ is the parallel (to the equilibrium magnetic field) velocity, $M$ is the magnetic moment and $\Omega_{H}$ is EP cyclotron frequency. This choice results in $\rho_{H} / a=0.01$ and $v_{H} / v_{A 0}=1.80$. Here, $\rho_{H} \equiv v_{H} / \Omega_{H}$ is the EP Larmor radius, $v_{H} \equiv \sqrt{E_{0} / m_{H}}$ is the characteristic EP birth speed, and $v_{A 0}$ is the on-axis Alfvén speed. The EP pressure drive is controlled by $n_{H}$, the EP density radial profile. In order to study the Alfvénic fluctuations in the central as well as outer core regions, we assume two dif- ferent EP radial profiles, as shown in Fig. 3. The profile (a) is numerically fitted from the ITER reference scenario SC2 ${ }^{28}$ in analogy to Ref. 8: it is adopted here to study resonant excitations of Alfvénic fluctuations in the central core region. The broader profile (b) is assumed to be outward-shifted, and is used to study the linear stability in the outer core region. As overall normalization, and unless otherwise explicitly stated, we set EP on-axis density $n_{H 0} / n_{i 0}=3.0 \times 10^{-3}$ as a reference value, with $n_{H 0}$ and $n_{i 0}$ being the on-axis density of EPs and bulk ions, respectively. It corresponds to $\mathrm{EP}$ on-axis ratio of kinetic to magnetic pressures $\beta_{H 0} \simeq 1.08 \times 10^{-2}$, similar to that of the considered ITER scenario (see Ref. 8 for an in depth discussion of this point).

As we are focusing on the linear stability properties of the Alfvénic fluctuation spectrum, in our simulations, we analyze the evolution of a single toroidal mode number $n$, with $n$ ranging between $n=2$ and $n=10$. This corresponds to neglecting nonlinear modemode coupling among different toroidal mode numbers, which will be studied in a separate work. Poloidal harmonics are retained accordingly for each $n$ in the range of $\left[n q_{\min }, n q_{\max }\right]$.

\section{LINEAR STABILITY ANALYSIS: SHEAR ALFVÉN FLUCTUATION SPECTRUM IN DTT}

In this section, we examine the linear shear Alfvén fluctuation spectrum of the DTT reference scenario described in Sec. II. Consistent with the general features of the shear Alfvén continuum illustrated in Fig. 2(b), simulation results can be essentially divided into a central core region and an outer core region with distinct behaviors. EPs with density profiles of Fig. 3(a) and (b) are used for maximized drive in central and outer core regions, respectively.

\section{A. Central core region linear stability analysis}

As a reference case for the linear stability analysis in the central core region, a simulation case with $n=4$ and $m$ from 3 to 14 is investigated in greater detail in this subsection. Fig. 4 shows the radial structures of scalar potential fluctuations, as well as the corresponding power spectrum in the $(r, \omega)$ plane. The linear dynamics are characterized by real frequency $\omega_{\mathrm{r}, \mathrm{EP}} / \omega_{A 0} \simeq 0.08630$ and linear growth rate $\gamma_{L} / \omega_{A 0} \simeq 0.02946$, where $\omega_{A 0}=$ $v_{A 0} / R_{0}$ is the on-axis Alfvén frequency. The fluctuations exhibit clear characteristics of reverse shear Alfvén eigenmodes (RSAEs), as the modes are radially located in the vicinity of the $q_{\min }$ surface, and the frequency is close to the local RSAE accumulation point frequency $\omega_{\text {ap }}$. In order to further analyze the underlying fluctuations, we use external antenna ${ }^{42,43}$ to excite the eigenmode in the same equilibrium without EPs, and verify the eigenmode structures, real frequency and damping rate in the MHD 

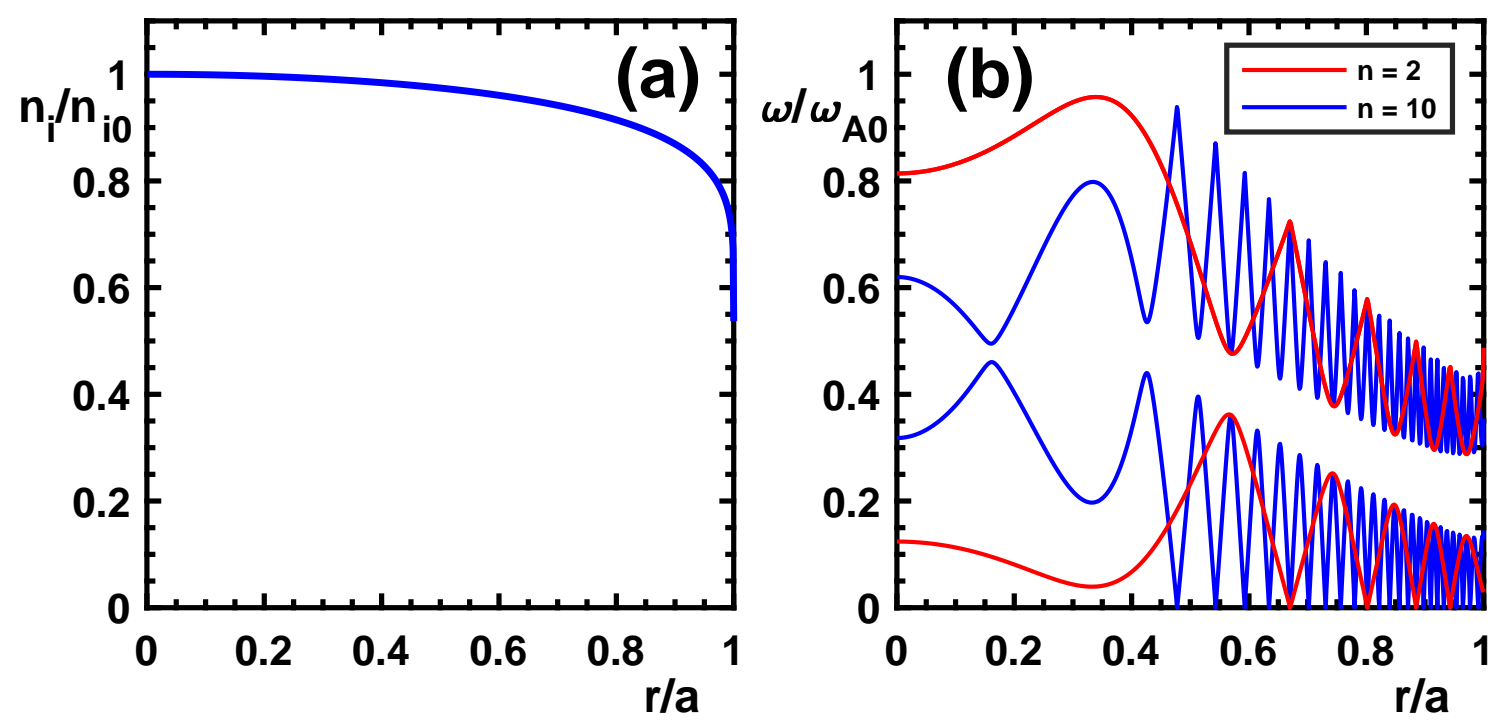

FIG. 2. Radial profile of bulk ion density $n_{i}$ normalized to its on-axis value $n_{i 0}$ (a) and the shear Alfvén continua for two typical mode numbers $n=2$ and $n=10$ (b).
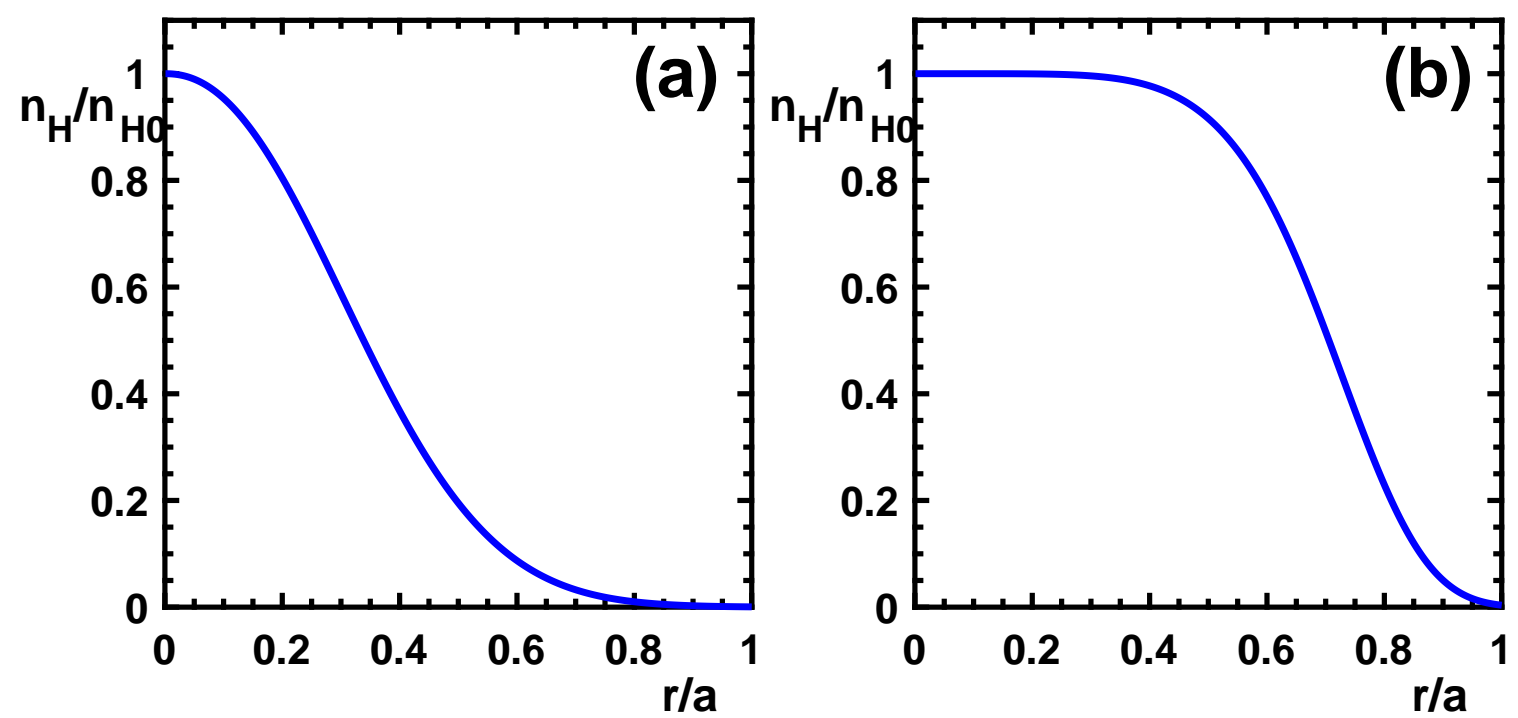

FIG. 3. EP density radial profiles $n_{H}$ normalized to its on-axis value $n_{H 0}$ used for: (a) analyzing Alfvénic fluctuation stability in the central core region; and (b), in the outer core region.

limit. Here, the antenna consists of an extrinsic driving source with fixed amplitude and tunable frequency. The fluctuation excited by antenna behaves as a forced oscillator, with the saturated response amplitude given by ${ }^{42,43}$

$$
\delta \varphi_{\mathrm{sat}} \propto \frac{1}{\sqrt{\left(\omega_{0}^{2}-\omega_{\mathrm{ant}}^{2}\right)^{2}+4 \gamma_{d}^{2} \omega_{\mathrm{ant}}^{2}}} .
$$

Here, $\delta \varphi_{\text {sat }}$ is the saturated scalar potential fluctuation amplitude, $\omega_{0}^{2}=\omega_{r}^{2}+\gamma_{d}^{2}$ with $\omega_{r}$ and $\gamma_{d}$ being, respectively, eigenmode real frequency and damping rate, and $\omega_{\text {ant }}$ is the antenna frequency. Fig. 5 shows $\delta \varphi_{\text {sat }}$ of $m=$ 4 harmonic from antenna frequency scan, from which the eigenmode frequency and damping rate can be obtained by fitting with Eq. (2), yielding $\omega_{\mathrm{r}, \mathrm{AE}} / \omega_{A 0} \simeq 0.06933$ and $\gamma_{d} / \omega_{A 0} \simeq 1.079 \times 10^{-3}$.

The clear upshift of the RSAE real frequencies in the presence of EPs suggests that their effect is nonperturbative. In order to illustrate the EP effect more clearly, we scan the EP density from the reference value $n_{H 0} / n_{i 0}=3.0 \times 10^{-3}$ down to $0.5 \times 10^{-3}$, and the obtained frequencies are shown in Fig. 6(a), along with the 

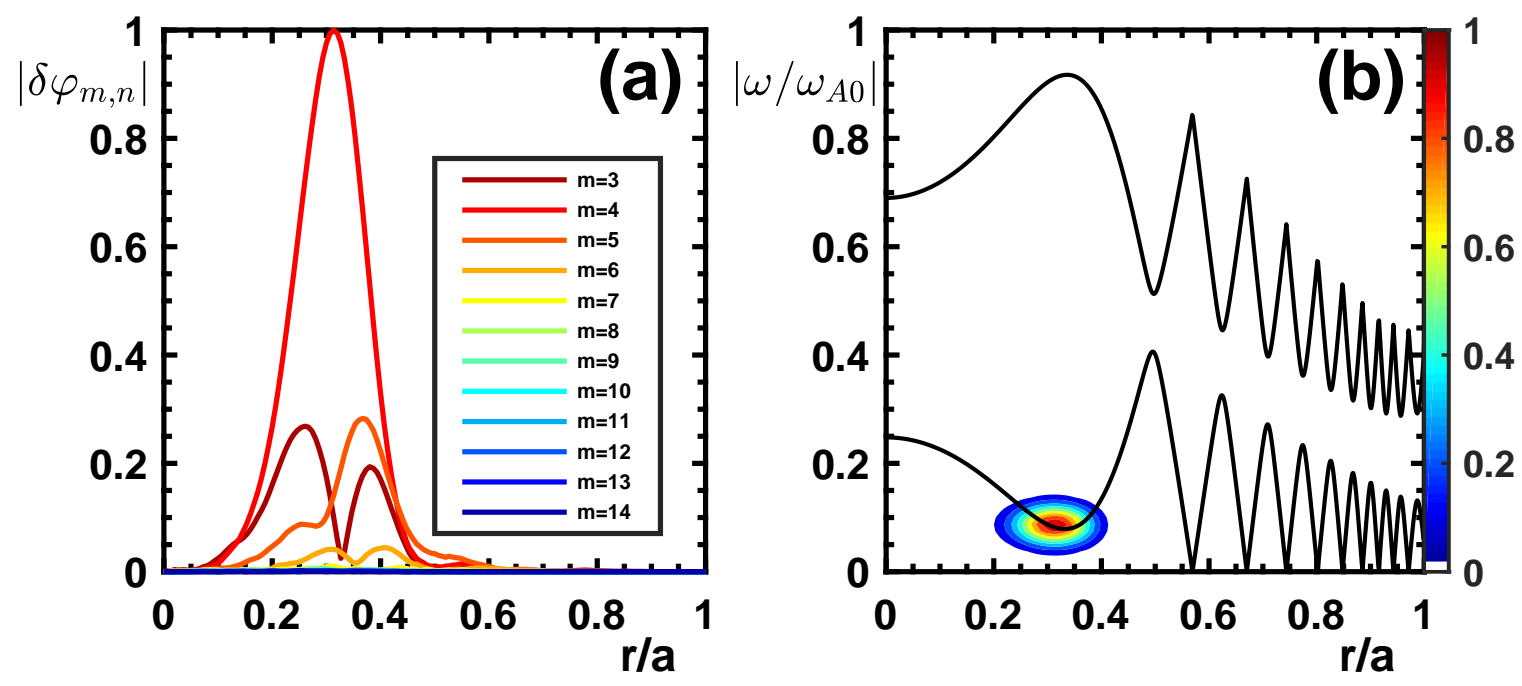

FIG. 4. Radial structures of each poloidal harmonics of scalar potential fluctuations (a) and the corresponding power spectrum in the $(r, \omega)$ plane (b) are shown in arbitrary units for the $n=4$ reference case in the central core region. The solid lines in figure (b) represent SAW continuum.

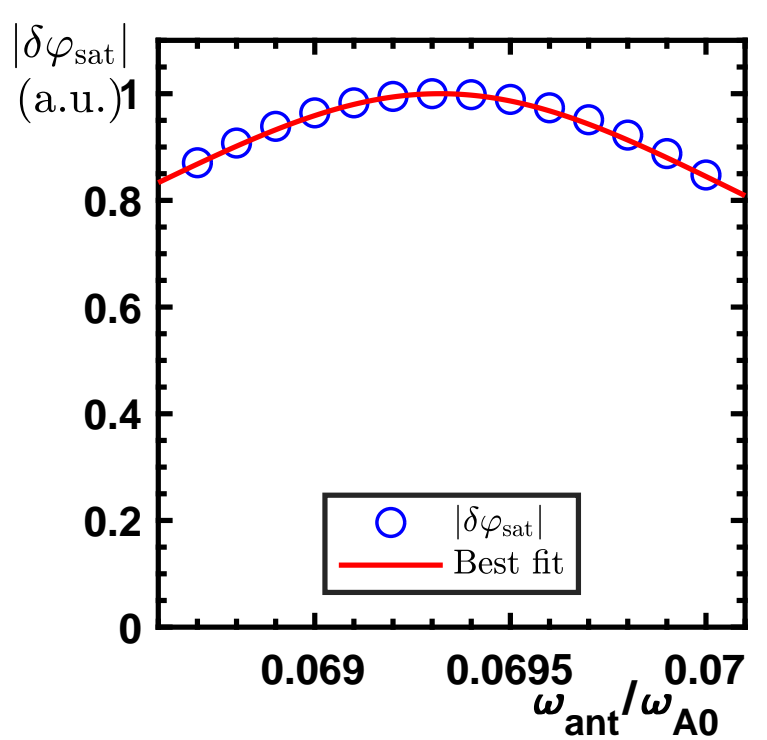

FIG. 5. Saturated response amplitudes $\delta \varphi_{\text {sat }}$ of $m=4$ harmonic with respect to antenna frequency $\omega_{\text {ant }}$ in the antenna frequency scan. The red curve corresponds to a best fit with $\omega_{\mathrm{r}, \mathrm{AE}} / \omega_{A 0} \simeq 0.06933$ and $\gamma_{d} / \omega_{A 0} \simeq 1.079 \times 10^{-3}$.

MHD limit $\omega_{\mathrm{r}, \mathrm{AE}}$ measured by antenna and the RSAE accumulation point frequency $\omega_{\text {ap }}$. The mode frequency $\omega_{r}$ is progressively upward shifted with increasing EP drive, and the modes move from well inside the frequency gap into the continuum. Therefore, for the $n=4$ reference case with $n_{H 0} / n_{i 0}=3.0 \times 10^{-3}$, the EP-induced frequency shift $\Delta \omega_{\mathrm{EP}} \equiv\left|\omega_{\mathrm{r}, \mathrm{EP}}-\omega_{\mathrm{r}, \mathrm{AE}}\right|$ is higher than the frequency mismatch of the eigenmode frequency with re- spect to the accumulation point $\Delta \omega_{\mathrm{SAW}} \equiv\left|\omega_{\mathrm{ap}}-\omega_{\mathrm{r}, \mathrm{AE}}\right|$, as labeled in Fig. 6(a). This confirms that the effect of EPs is clearly non-perturbative ${ }^{44}$. In fact, the underlying fluctuations are no longer "pure" RSAE gap modes. Instead, they are RSAEs strongly affected by EP kinetic response, and are in the parameter range characterizing their transition to EPMs. Moreover, the EP nonperturbative effect can also be seen from the damping rates, as shown in Fig. 6(b). Here, in the EP driven simulation cases, the damping rates are estimated as $\gamma_{d}=\gamma_{\text {drive }}-\gamma_{L}$, where $\gamma_{\text {drive }}$ stands for "driving rate", calculated from the instantaneous wave-particle power exchange, integrated over the particle phase space and weighted by the sum of kinetic and magnetic energy density $^{22}$. The significant increase of $\gamma_{d}$ with increasing EP drive suggests a possible change of the dominant damping mechanism, and the important role played by SAW continuum. In all the cases, radiative damping ${ }^{45}$ remains important since $\gamma_{d} \lesssim\left|\Delta \omega_{\mathrm{SAW}}\right| \lesssim\left|\Delta \omega_{\mathrm{EP}}\right|$ as shown in Fig. 6.

More insights into the wave-particle interactions underlying mode destabilization can be obtained from the analysis of the wave-particle power exchange as well as the EP resonance condition. We focus on the waveparticle power density transfer, which is averaged over poloidal $(\theta)$ and toroidal angles $(\phi)$ and reported in the reduced phase space $\left(r_{0}, M, U_{0}\right)^{22}$. Note that, $r_{0}$ and $U_{0}$ indicate the value of $r$ at which each particle crosses the equatorial plane at $\theta=0$, and the corresponding value of $U$. Fig. 7 shows $\left.<P\left(r_{0}, M, U_{0}\right)\right\rangle_{\Delta V}$ integrated over a radial shell of volume $\Delta V$ around the mode localization for the $n=4$ reference case, with the solid and dashed lines indicating, respectively, the trapped- 

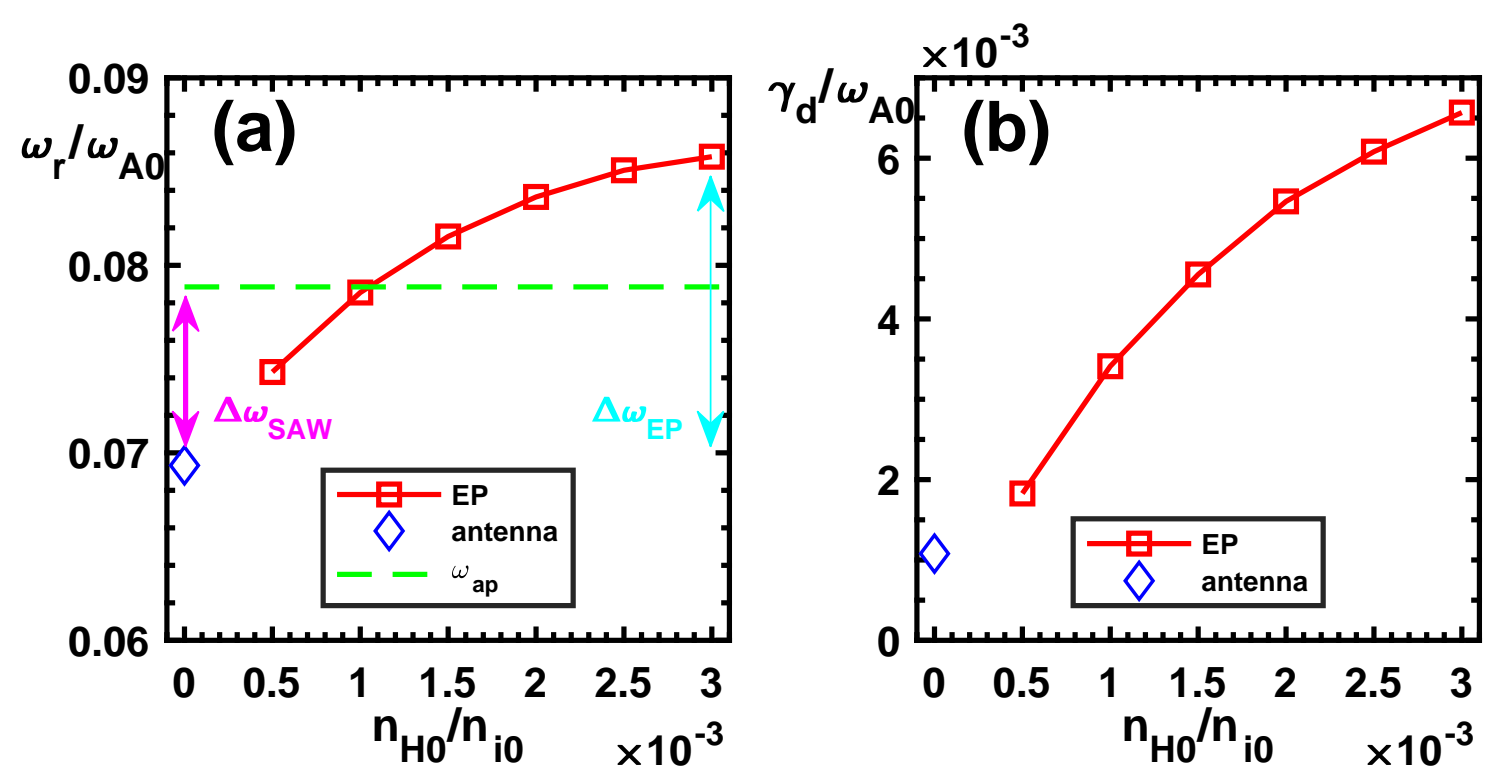

FIG. 6. RSAE real frequencies $\omega_{r}$ (a) and damping rates $\gamma_{d}$ (b) from the EP density scan, the respective values in the MHD limit $\left(n_{H 0} / n_{i 0}=0\right)$ measured by antenna are also included. In addition, the RSAE accumulation point $\omega_{\text {ap }}$ is shown as a green dashed line in figure (a).

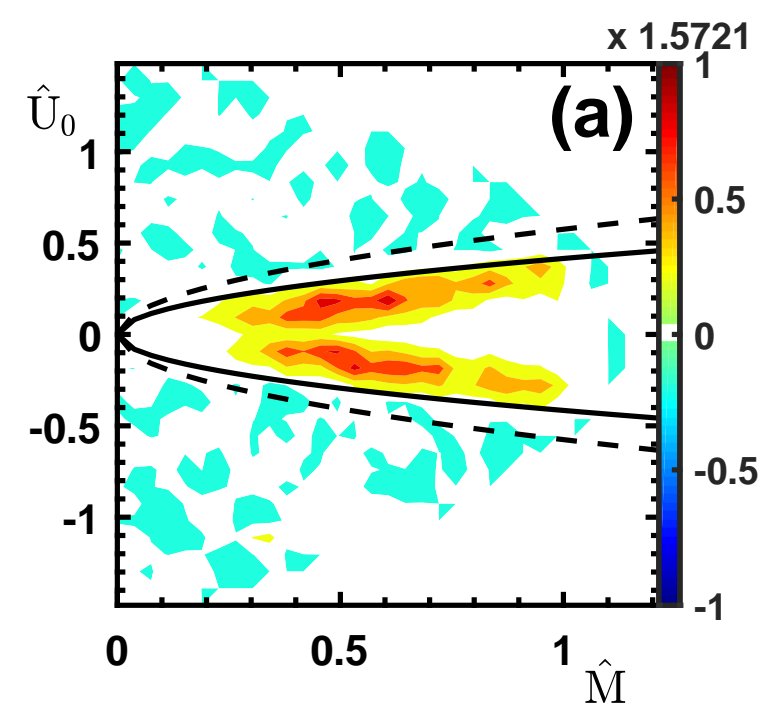

FIG. 7. For the $n=4$ reference case, wave-particle power transfer density $<P\left(r_{0}, M, U_{0}\right)>_{\Delta V}$, integrated over a radial shell of $0.24 \leq r_{0} / a \leq 0.46$, is shown in the normalized $\left(\hat{M}, \hat{U}_{0}\right)$ plane, with $M$ in units of $E_{0} / \Omega_{H 0}\left(\Omega_{H 0}\right.$ is on-axis cyclotron frequency) and $U_{0}$ in units of $v_{H}$. Positive sign represents power transfer from particles to the wave. Solid and dashed lines indicate approximately inner and outer trappedpassing EP boundaries of the considered radial shell.

passing EP boundaries of the inner and outer radial limits. We can observe that the mode drive is mostly provided by magnetically trapped particles. The fact that the underlying fluctuations are characterized by single $n$ and constant frequency allows us to use test particles to identify the structure of wave-particle resonances. Following Refs. 22, 44, and 46, we can take the extended phase space Hamiltonian $C \equiv \omega P_{\phi}-n E$ as constant of the perturbed motion in addition to the conserved magnetic moment $M$. Here, $P_{\phi}$ is the toroidal angular momentum, which, at the leading order, can be written as $P_{\phi} \simeq m_{H} R U+e_{H} R_{0}\left(\Psi_{\text {eq }}-\Psi_{\text {eq0 }}\right) / c$, where $R$ is the major radius coordinate, $\Psi_{\text {eq }}$ is the equilibrium flux function defined by the form of the confining magnetic field, $\mathbf{B} \equiv R_{0} B_{\phi 0} \nabla \phi+R_{0} \nabla \Psi_{\mathrm{eq}} \times \nabla \phi$, and $\Psi_{\text {eq0 }}$ is its on-axis value. Then, the particle phase space can be divided into "slices" characterized by different values of $M$ and $C$, whose linear and nonlinear dynamics are independent of each other. Meanwhile, the wave-particle power exchange can be described as the sum of contributions from all the slices. We can then focus on a single slice $\left(M_{0}, C_{0}\right)$, where wave-particle power transfer is significant, identified from plots like Fig. 7. By selecting a set of test particles characterized by $M=M_{0}, C=C_{0}$, and with $U_{0}$ computed correspondingly as $U_{0}=U\left(r_{0}, M_{0}, C_{0}\right)$, the characteristic dynamic frequencies, such as precession frequency $\omega_{d}$ and bounce/transit frequency $\omega_{b}$ for trapped/passing particles, can then be computed by following the corresponding equilibrium orbits. The resonance frequency $\omega_{\text {res }}$ for trapped particles is given $a^{44}$

$$
\omega_{\mathrm{res}}\left(r_{0}, M_{0}, C_{0}, \ell\right)=n \omega_{d}+\ell \omega_{b},
$$

where $\omega_{d}=(\Delta \phi / 2 \pi-\sigma \bar{q}) \omega_{b}, \omega_{b}=2 \pi / \tau_{b}$, and $\ell$ is the "bounce" harmonic. Here, $\Delta \phi$ is the change of toroidal angle $\phi$ over a full bounce time $\tau_{b}=\oint d \theta / \dot{\theta}, \sigma=\operatorname{sgn}(U)$, and $\bar{q}$ is the average safety factor integrated along the particle orbit ${ }^{44}$. For passing particles, meanwhile, $\omega_{\text {res }}$ is 


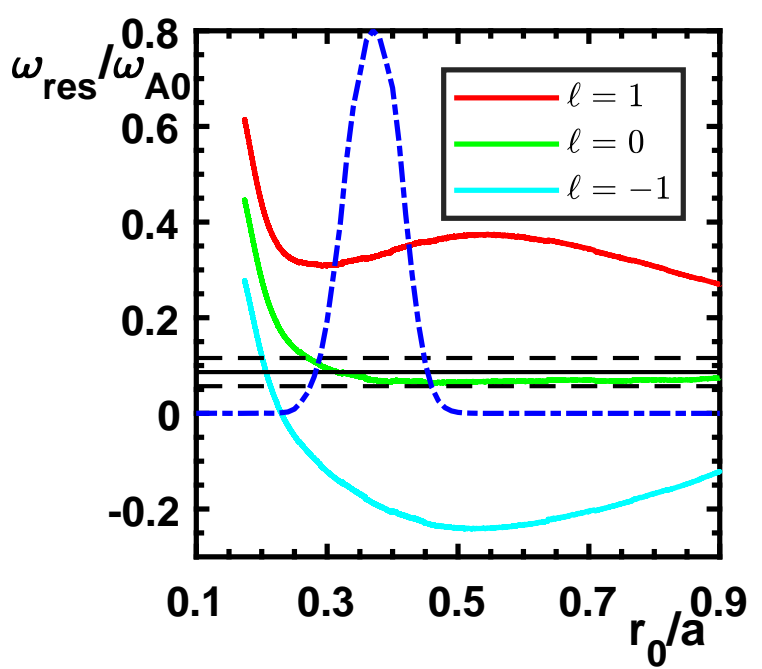

FIG. 8. For the $n=4$ reference case, $\omega_{\text {res }}\left(r_{0}\right)$ with several bounce harmonics $\ell$ calculated from test particles orbits as specified in the text. The black solid line indicates $\omega_{r}$ of the same case, and the dashed lines stand for $\omega_{r}+\gamma_{L}$ and $\omega_{r}-\gamma_{L}$, respectively, corresponding to the criterion of Eq. (5). The over-imposed blue dash-dotted curve represents the radial dependence of power density exchange of the test particles in arbitrary units. The dominant bounce harmonic is $\ell=0$.

given by

$$
\omega_{\mathrm{res}}\left(r_{0}, M_{0}, C_{0}, \ell\right)=n \omega_{d}+\ell \omega_{b}+(n \bar{q}-m) \sigma \omega_{b}
$$

For a mode with linear growth rate $\gamma_{L}$, Eqs. (3) and (4) allow us to express the condition for significant waveparticle power exchange to occur:

$$
\left|\omega-\omega_{\text {res }}\left(r_{0}, M_{0}, C_{0}, \ell\right)\right| \lesssim \gamma_{L}
$$

For the $n=4$ reference case, we select $M_{0} \Omega_{H 0} / E_{0}=$ 0.4933 and $C_{0} / E_{0}=-1.955$, corresponding to $U_{0} / v_{H}=$ 0.1852 at $r_{0} / a=0.3600$, that is, a resonance peak for trapped particles. Test particles are distributed in the radial interval $0.18 \leq r_{0} / a \leq 0.95$, and Fig. 8 shows $\omega_{\text {res }}\left(r_{0}\right)$ with several bounce harmonics. From the criterion given in Eq. (5), the dominant resonance is clearly the precession resonance with $\ell=0$.

Similar to the $n=4$ reference case analyzed above, we analyze the linear stability properties and SAW fluctuation spectrum in the central core region by means of EPdriven simulations, antenna excitations and test particle methods with $n$ in the range $2 \leq n \leq 10$. All EP-driven simulations are found to be strongly unstable and the fluctuations are characterized by RSAE mode structures, similar to the ones of the $n=4$ reference case shown in Fig. 4. Moreover, by evolving test particles in each simulations, we find that the drive of these RSAE fluctuations is predominantly provided by the precession resonances

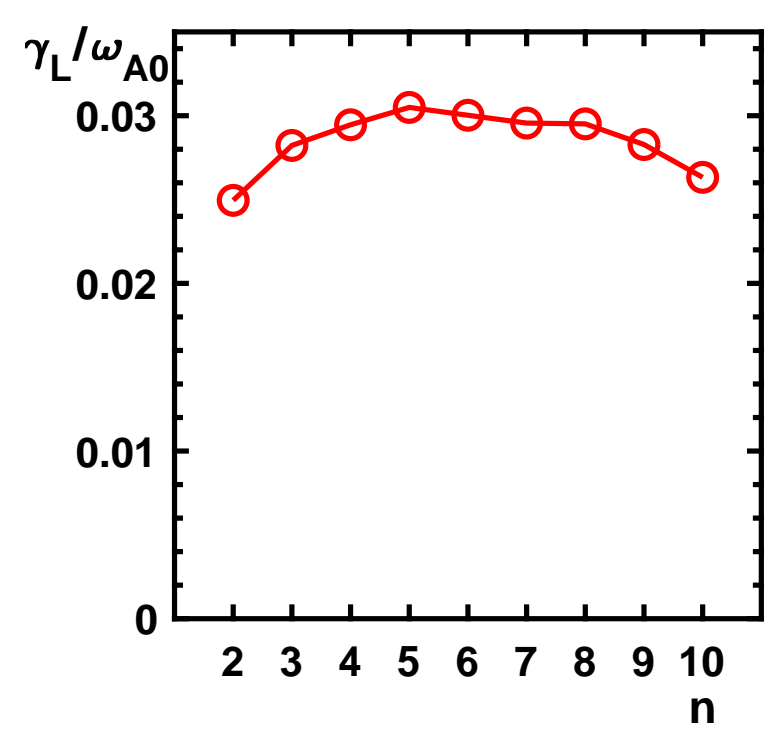

FIG. 9. Linear growth rates $\gamma_{L}$ of the RSAE fluctuations with $n$ in the range $2 \leq n \leq 10$.

of trapped particles for all considered toroidal mode numbers. The linear stability properties are summarized in Fig. 9, for the reference value $n_{H 0} / n_{i 0}=3.0 \times 10^{-3}$ of EP density. All considered mode numbers are characterized by similar linear growth rates, and the growth rate is quite insensitive to $n$. Thus, we could reasonably conclude that, the most unstable mode number of the RSAE fluctuations in the central core region is of the order of $n \sim O(10)$ as expected ${ }^{8,9}$. This is quite different from SAW fluctuations in present day tokamaks, where the most unstable mode number is generally of the order of $n \sim O(1)^{8,9}$. The most unstable mode number found in our simulations can be verified against theoretical estimations ${ }^{17,47-50}$ that the perpendicular (to the equilibrium magnetic field) wavelength of the underlying fluctuations is comparable with the characteristic particle orbit width, i.e., $k_{\perp} \rho_{d} \simeq 1$, where $k_{\perp}$ is the perpendicular wave number and $\rho_{d}$ is the resonant EP magnetic drift orbit width. Interested readers may refer to Refs. 17, 4750 for a detailed discussion. Fig. 10 shows typical values of $k_{\perp} \rho_{d}$ with respect to $n$, where $\rho_{d}$ is the banana orbit width of resonant trapped particles. We can see that the criterion $k_{\perp} \rho_{d} \simeq 1$ is satisfactorily verified for the most unstable mode number in our simulations. Indeed, the most unstable mode number being $n \gg O(1)$ is exactly what we expect for DTT as well as other next generation tokamaks, in which EPs are generally characterized by smaller dimensionless orbits $\left(\rho_{H} / a=0.01, \rho_{d} / a \simeq 0.035\right.$ for the present case) than in present day devices. The present results also suggest the important role played by meso-scale dynamics in the nonlinear regime, which are beyond the scope of the present analysis and will be investigated in a future work.

The real frequencies of RSAE fluctuations in EP-driven 


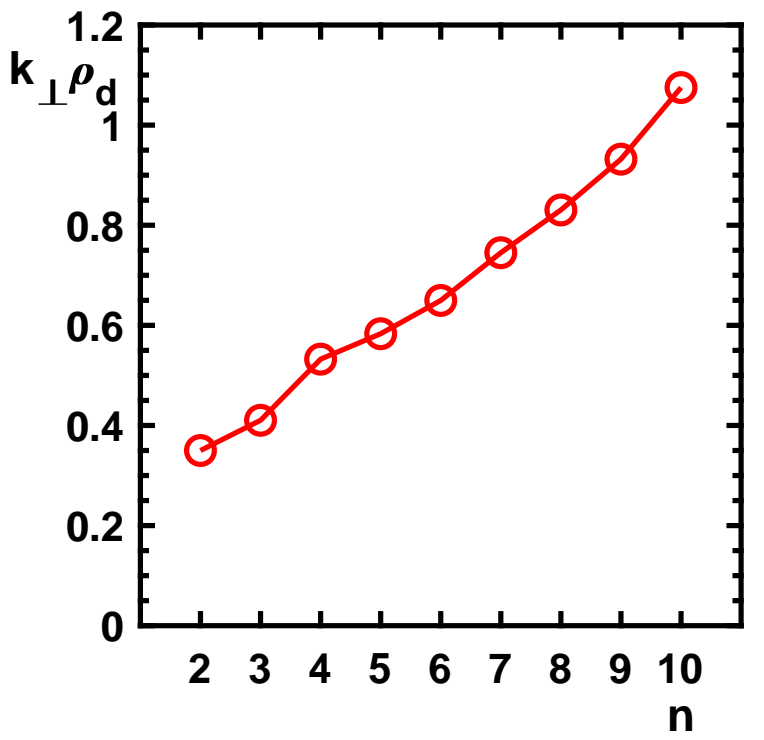

FIG. 10. Numerical calculation of $k_{\perp} \rho_{d}$ with respect to $n$. Here, $k_{\perp}$ is calculated as $k_{\perp}^{2}=k_{r}^{2}+k_{\theta}^{2}$, where $k_{r}$ and $k_{\theta}$ are estimated as $k_{r}=1 / \Delta r$ and $k_{\theta}=m / r_{\max }$ from the mode structures: $\Delta r$ is the radial width of the mode structure envelope at half maximum amplitude, and $r_{\max }$ is the radial coordinate at maximum amplitude. $\rho_{d}$ is measured by the orbits of resonant test particles.

simulations and the eigenmode real frequencies measured by antenna excitations are shown in Fig. 11 as a function of the toroidal mode number $n$. We note that the EPinduced frequency shifts $\Delta \omega_{\mathrm{EP}}$ (defined in Fig. 6) are comparable for every toroidal mode number, consistent with their similar growth rates (see Fig. 9) and, thus, similar EP drives. However, significant changes of mode dynamics with $n$ can be observed and understood when comparing mode frequency to the corresponding RSAE accumulation point frequency $\omega_{\text {ap }}$. In the lower mode number limit, the modes reside inside the continuum and $\mathrm{EP}$ effects are clearly non-perturbative since $\Delta \omega_{\mathrm{EP}} \gtrsim$ $\Delta \omega_{\mathrm{SAW}}{ }^{44}$. In the higher mode number limit, however, the modes can be separate from the continuum, and the EP effects could therefore be considered perturbative.

\section{B. Outer core region linear stability analysis}

Following the same approach as in Sec. III A, a simulation case with $n=6$ and $m$ in the range of 5 to 21 is presented first as a reference case for the outer core region. The radial mode structures and the corresponding power spectrum are shown in Fig. 12. The linear dynamics are characterized by $\omega_{\mathrm{r}, \mathrm{EP}} / \omega_{A 0} \simeq 0.3331$ and $\gamma_{L} / \omega_{A 0} \simeq 0.01585$, and the underlying fluctuation can be recognized as toroidal Alfvén eigenmode (TAE), since the mode frequency lies inside the toroidicity induced frequency gaps, and several coupled poloidal harmonics contribute to the mode structure. The eigenmode real

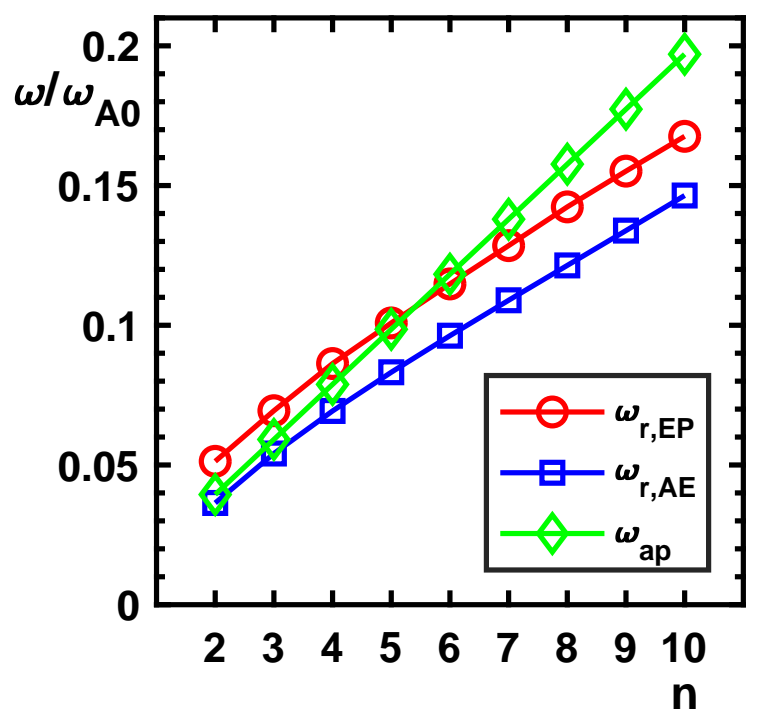

FIG. 11. The real frequencies of the RSAE fluctuations driven by EPs $\omega_{\text {r,EP }}$ are shown in red circles as a function of the toroidal mode number $n$. Eigenmode real frequencies $\omega_{\mathrm{r}, \mathrm{AE}}$ measured by antenna excitations are shown in blue squares. Corresponding RSAE accumulation points $\omega_{\text {ap }}$ are shown in green diamonds.

frequency and damping rate in the MHD limit can be measured by antenna frequency scans, and are given by $\omega_{\mathrm{r}, \mathrm{AE}} / \omega_{A 0} \simeq 0.3615$ and $\gamma_{d} / \omega_{A 0} \simeq 0.07902$. Note that the TAE fluctuations are more strongly damped than the RSAEs in the central core region. This could be due to the narrower toroidicity induced gap, and thus, stronger TAE coupling to SAW continuum due to the artificial $\epsilon=0.18$ adopted in our simulations. Based on the mode structure, we can conclude that the dissipation is predominantly due to continuum damping ${ }^{51,52}$, unlike the RSAE case characterizing the central core region (cf. Sec. III A) dominated by radiative damping. However, the qualitative features of mode structures and resonant excitation by EPs are unaltered, since they predominantly depend on $q$ and EP radial profiles ${ }^{9,18,19}$. Fig. 13(a) shows $\omega_{r}$ as a function of $n_{H 0} / n_{i 0}$ in a EP density scan. The mode frequency in the MHD limit without EPs is also shown as measured by antenna excitation. We can observe that, despite the frequency gap widths are reduced in our model description with $\epsilon=0.18$, $\Delta \omega_{\mathrm{EP}}<\Delta \omega_{\mathrm{SAW}}$ can still be satisfied for the reference case and, thus, the EP effect could be considered perturbative. In fact, if we assume perturbative EP effects, i.e., $\gamma_{L}$ scales linearly with $n_{H 0}$, we are able to extrapolate $\gamma_{L}$ in the EP density scan to the MHD limit without EPs, and verify the extrapolated $\gamma_{d}$ against the damping rate given by antenna excitation. As shown in Fig. 13(b), the agreement of two methods is quite satisfactory. Moreover, the extrapolation of $\gamma_{L}$ also suggests an excitation threshold for TAE onset being approximately given by $n_{H 0} / n_{i 0} \simeq 2.48 \times 10^{-3}$ for the $n=6$ case. 

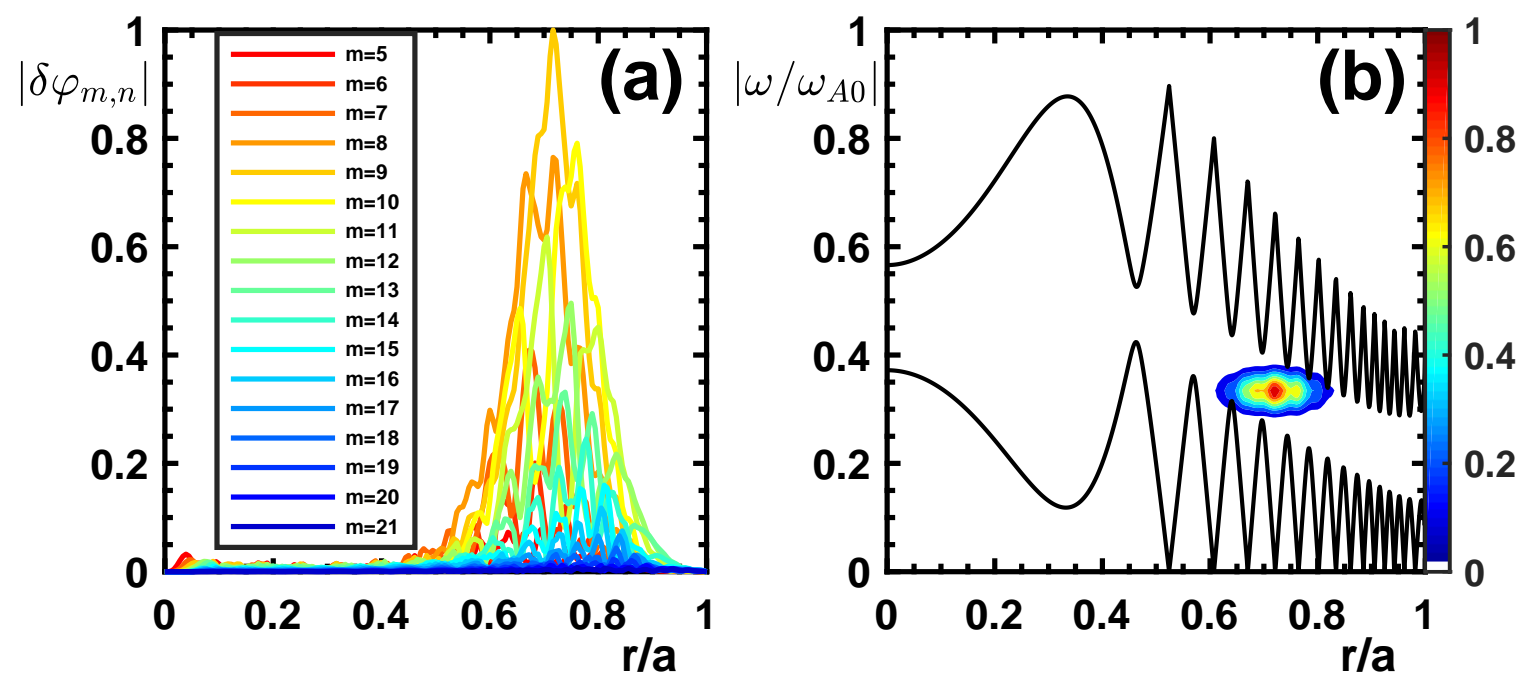

FIG. 12. Analogous to Fig. 4, radial structures of each poloidal harmonics of scalar potential fluctuations (a) and the corresponding power spectrum in the $(r, \omega)$ plane $(\mathrm{b})$ are shown in arbitrary units for the $n=6$ reference case in the outer core region. The solid lines in figure (b) represent the SAW continuum.
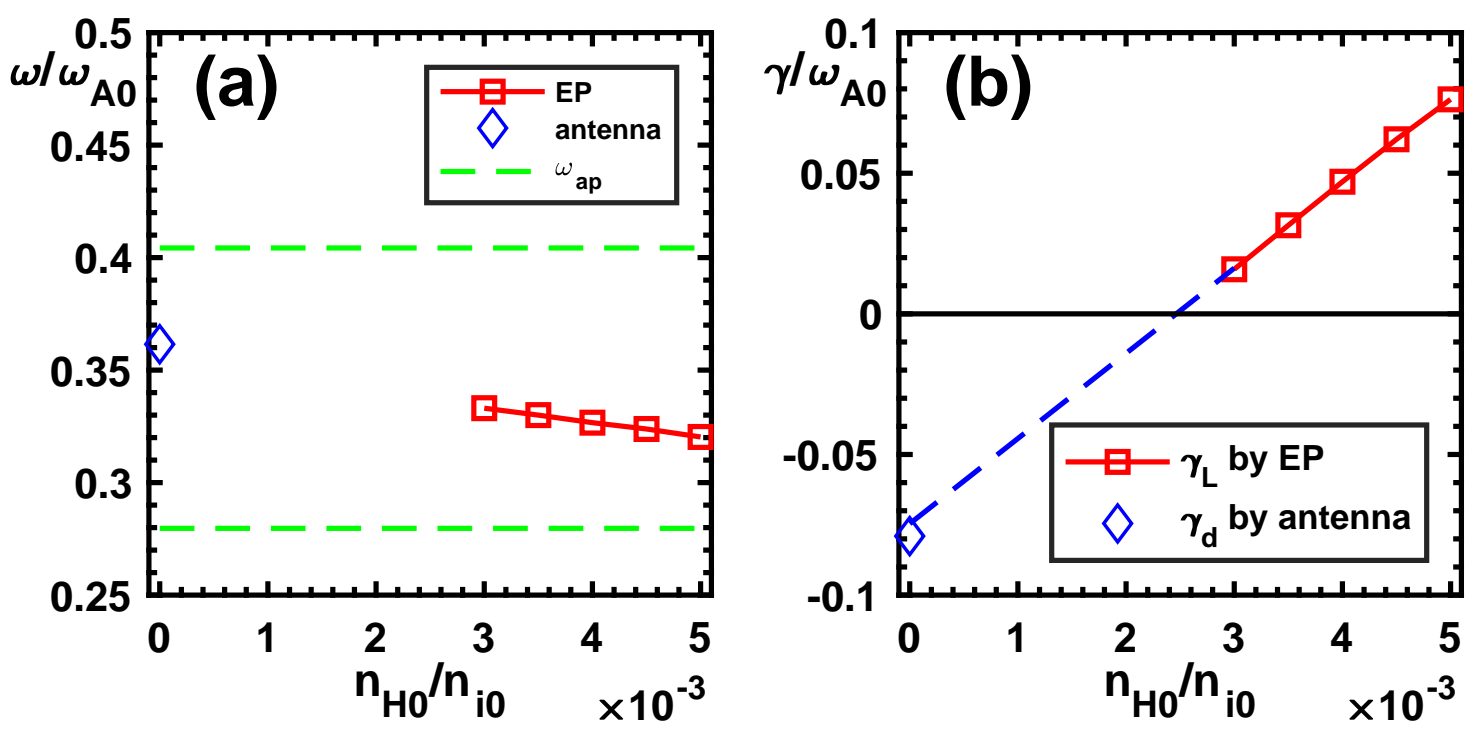

FIG. 13. TAE real frequencies $\omega_{\mathrm{r}, \mathrm{EP}}(\mathrm{a})$ and linear growth rates $\gamma_{L}$ (b) from the EP density scan are shown, $\omega_{\mathrm{r}, \mathrm{AE}}$ and $\gamma_{d}$ in the MHD limit measured by antenna are also included in respective frames. In addition, the upper and lower TAE accumulation points $\omega_{\text {ap }}$ are shown as the green dashed lines in figure (a).

For the $n=6$ reference case, Fig. 14(a) shows the wave-particle power density transfer $<$ $P\left(r_{0}, M, U_{0}\right)>_{\Delta V}$ integrated over a radial shell $\Delta V$ around mode location; and in Fig. 14(b), the radial profiles of power density transfer integrated over particle velocity space $<P\left(r_{0}, M, U_{0}\right)>_{M, U_{0}}$ are shown for trapped, counter-passing and co-passing particles, respectively. We can observe that, in general, trapped and passing particles play comparable roles in the mode drive. Trapped particle response is more localized in the $\left(M, U_{0}\right)$ plane (Fig. 14(a)), while passing particles are characterized by sharper radial profiles around several radial locations (Fig. 14(b)). The different features of trapped and passing particles can be further illustrated by test particles chosen from power transfer peaks of each type. Fig. 15 shows $\omega_{\text {res }}\left(r_{0}\right)$ with multiple bounce/transit harmonics $\ell$, for trapped, counterand co-passing particles. Here, a representative poloidal harmonic $m=10$ is used for passing particles resonance frequencies. We can observe that the relevant bounce 

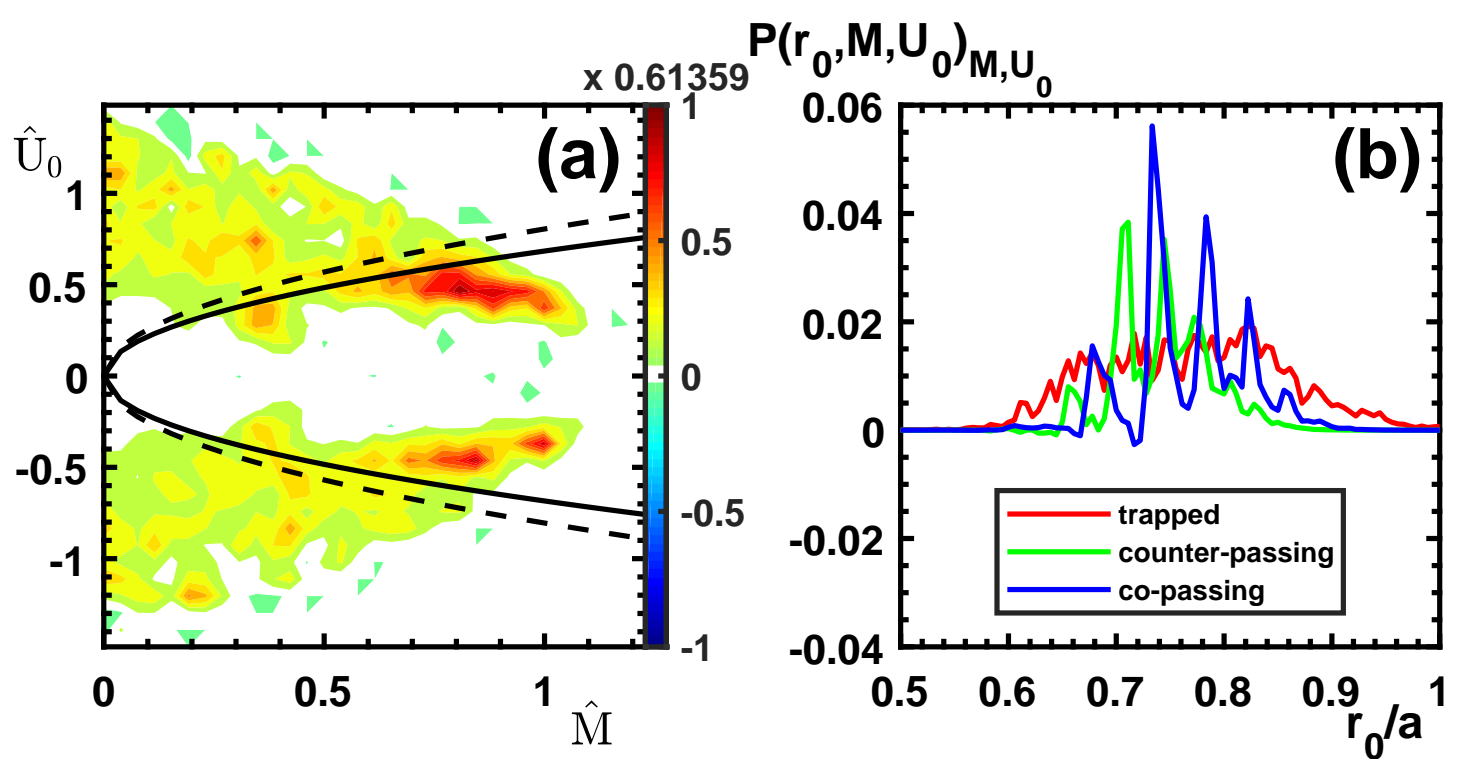

FIG. 14. The wave-particle power density transfer $<P\left(r_{0}, M, U_{0}\right)>_{\Delta V}$ integrated over a radial shell of $0.65 \leq r_{0} / a \leq 0.9$ (a). Normalization of $\left(\hat{M}, \hat{U}_{0}\right)$ axes is the same as in Fig. 7. The solid and dashed lines indicate approximately the trapped-passing EP boundaries of inner and outer limits of the considered radial shell. In frame (b), the radial profile of $<P\left(r_{0}, M, U_{0}\right)>_{M, U_{0}}$ integrated over $M$ and $U_{0}$ coordinates, is shown for trapped, counter-passing and co-passing particles, respectively.
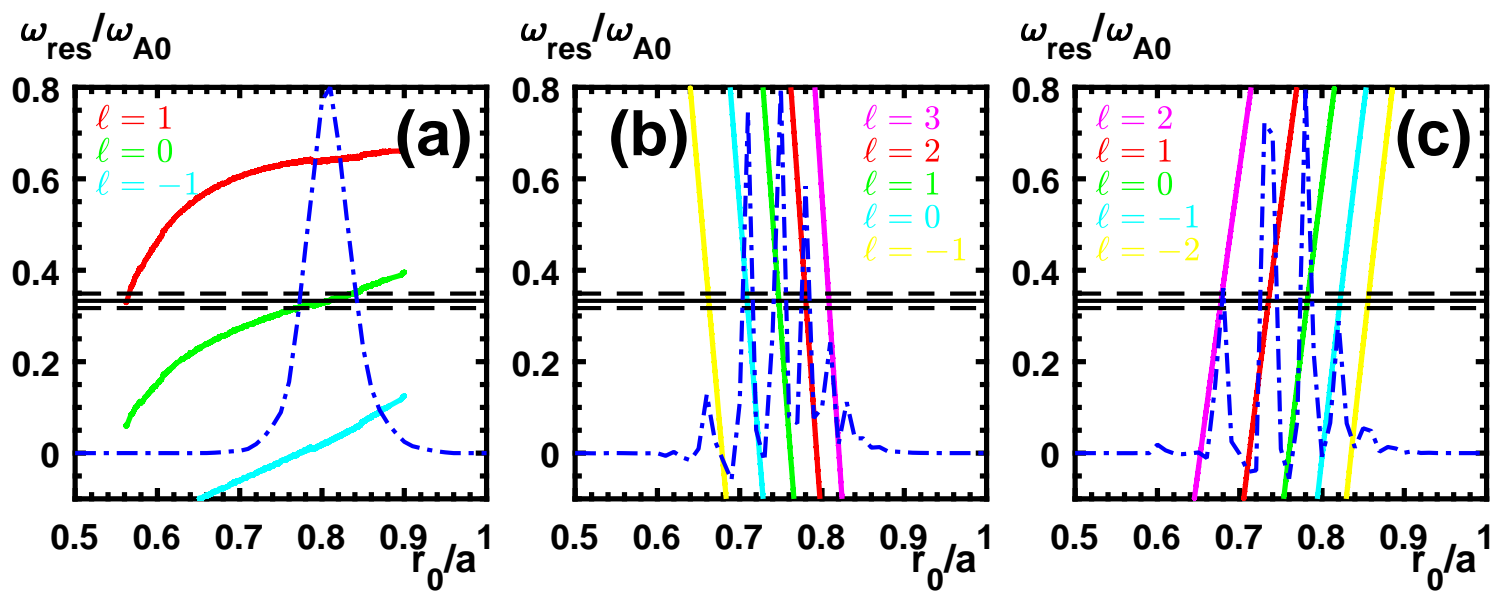

FIG. 15. $\omega_{\text {res }}\left(r_{0}\right)$ calculated from respective test particles orbits for trapped particles (a), counter-passing particles (b) and co-passing particles (c). A typical poloidal harmonic $m=10$ is used for figure (b) and (c). The black solid line indicates $\omega_{r}$ and the dashed lines show, respectively, $\omega_{r}+\gamma_{L}$ and $\omega_{r}-\gamma_{L}$. The over-imposed blue dash-dotted curve represents the radial dependence of power exchange of the test particles in arbitrary units.

harmonic for trapped particle resonance is $\ell=0$, similar to the cases of the RSAE fluctuations in the central core region. For passing particle resonances, a broad range of transit harmonics are involved, and multiple poloidal harmonics are excited consistently, as expected from the passing-particle resonance condition given in Eq. (4). Moreover, passing particles show much steeper $\omega_{\text {res }}\left(r_{0}\right)$ profiles than trapped particles due to the $\propto(n \bar{q}-m) \sigma \omega_{b}$ term in Eq. (4), which dominates the radial variation of $\omega_{\text {res }}\left(r_{0}\right)$, noting that $n q^{\prime} r=n r(d q / d r)$ and $\omega_{b} / \omega_{r}$ are both large for the present case. Thus, for given poloidal mode number, different passing particle transit harmonic resonances take place at different radial positions where $\omega_{\text {res }}\left(\bar{q}\left(r_{0}\right), \ell\right) \simeq \omega_{r}$, with much shorter individual resonance width $\left(\sim O\left(\gamma_{L} /\left(n q^{\prime} \omega_{b}\right)\right)\right)$ than that of trapped particles. The structures of $<P\left(r_{0}, M, U_{0}\right)>_{M, U_{0}}$ shown Fig. 14(b) are, thus, the result of different features of trapped and passing particle resonance frequencies shown in Fig. 15, consistent with the analysis of Refs. 9 and 44. It is worthwhile to point out that $\omega_{\text {res }}\left(r_{0}\right)$, shown in Fig. 15 , only reflect a single slice of phase space characterized by constant $\left(M_{0}, C_{0}\right)$; however, $<P\left(r_{0}, M, U_{0}\right)>_{M, U_{0}}$ 


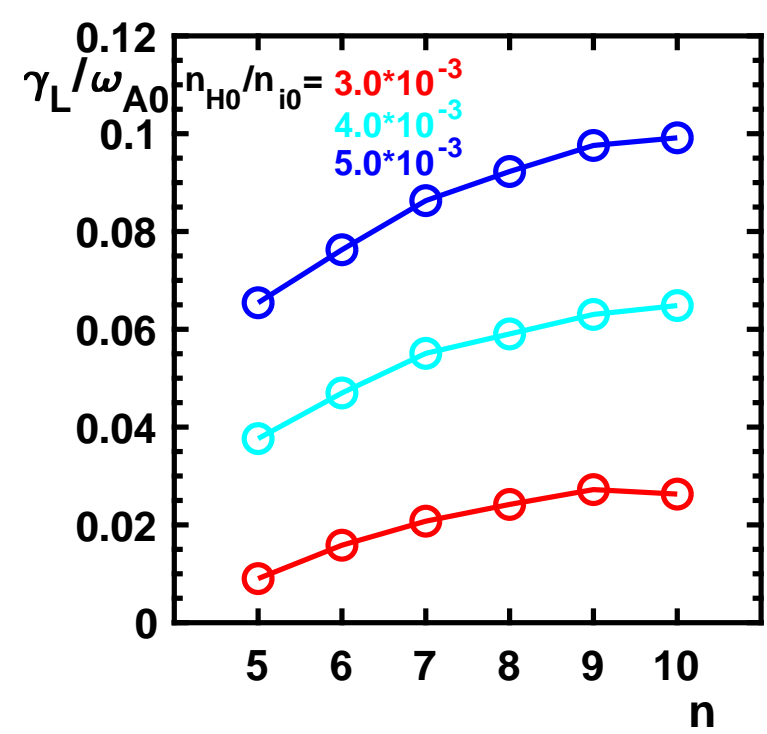

FIG. 16. Linear growth rates $\gamma_{L}$ of the TAE fluctuations with $n$ in the range of $5 \leq n \leq 10$. Three EP densities are used with $n_{H 0} / n_{i 0}=3.0, \overline{4} .0,5.0 \times 10^{-3}$.

shown in Fig. 14(b) consist of contributions from the whole phase space. Furthermore, from the linear resonance conditions and wave-EP power transfer, we can conjecture that trapped particles will behave more coherently in the fluctuation-induced transport process, while passing particles transport will be more diffusive $e^{9,26,44}$.

Similar studies are extended to other toroidal modes for the analysis of linear stability properties of the outer core region, as we did in Sec. III A for the central core region. The antenna excitation results are not shown here, since the TAEs can be relatively strongly coupled with the SAW continua and, therefore, it is not always possible to separate the eigenmode structures from the responses of the SAW continuum. As a matter of fact, the clearest response to antenna excitations and the best agreement between antenna excitations and EP-driven simulations are achieved for $n=6$. This is shown in Fig. 13, where the TAE mode frequency lies in the middle of the gap and, thus, the couplings with upper and lower SAW continua are minimized. As a survey of the $\mathrm{AE}$ linear stability properties in the outer core region, EP-driven simulations with three reference densities at $n_{H 0} / n_{i 0}=3.0,4.0,5.0 \times 10^{-3}$ for toroidal mode numbers in the range of $5 \leq n \leq 10$ are presented. Cases with $n$ lower than 5 are stable or marginally unstable in the weakest drive simulations due to small drift orbits and diamagnetic drift frequencies, and are consequently far from the most unstable condition. The linear growth rates of the TAE fluctuations with respect to $n$ for the three EP reference densities are shown in Fig. 16. These results suggest that the most unstable mode number is $n \gtrsim 10$ for the TAE fluctuations in the outer core region, slightly higher than the RSAE fluctuations in the cen-

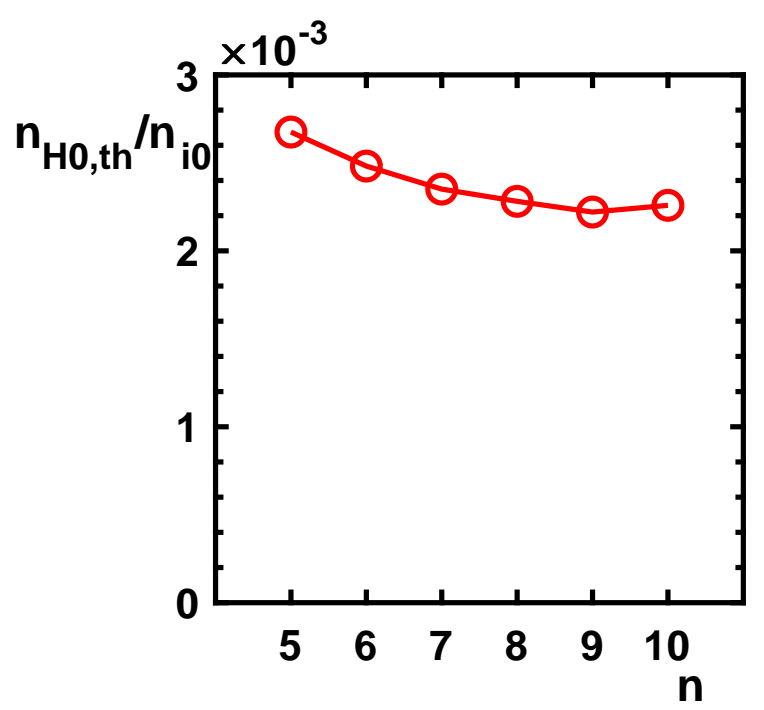

FIG. 17. TAE excitation threshold $n_{\mathrm{H} 0 \text {,th }}$ estimated by extrapolating $\gamma_{L}$ from the results considered in Fig. 16.

tral core region, as expected from analytical theory ${ }^{9,18,19}$. The EP concentration threshold for mode excitation can be estimated by assuming a linear scaling of $\gamma_{L}$ with respect to $n_{H 0}$, and its dependence on $n$ is shown in Fig. 17 , which further confirms that the most unstable mode number occurs for $n \gtrsim 10$.

From the wave-particle power exchange and test particle evolution, the resonance conditions of the weakest drive TAE fluctuations (with $n_{H 0} / n_{i 0}=3.0 \times 10^{-3}$ ) are similar to the $n=6$ reference case for all considered toroidal mode numbers. That is, precession resonance for trapped particles, and transit harmonic resonance for passing particles, with the two types of particles playing comparable roles in the overall power exchange. The most unstable mode number can be determined by maximization of the combined contribution from trapped and passing particles, which is expected to be in-between the most unstable modes for each type of particles, estimated based on $k_{\perp} \rho_{d} \sim O(1)$. Fig. 18 shows typical values of $k_{\perp} \rho_{d}$ calculated with respect to $n$ for trapped, counterand co-passing particles. We can conclude that the most unstable mode number excited by trapped EP resonances is $n \sim O(10)$, while the one by passing EP resonances is $n>10$. The most unstable mode number found in our simulations lies in between the two limits, consistent with our analysis. Note that, in our simulations, EPs are assumed to be isotropic fusion produced alpha particles; thus, the characteristic trapped particle banana orbit width and/or passing particle drift orbit width $\rho_{d}$ reflect the high birth energy. However, in a realistic DTT operation scenario, EPs could be very likely produced by (negative) neutral beam injection in the outer core region, with lower energy than fusion alphas, and/or ion cyclotron resonance heating in the central core region. 


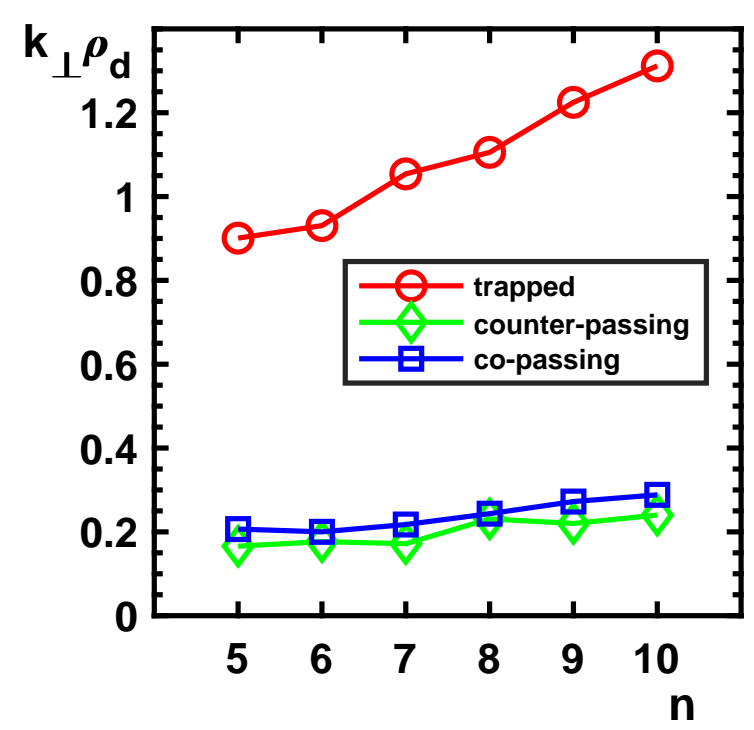

FIG. 18. Numerical calculations of $k_{\perp} \rho_{d}$ with respect to $n$, with $\rho_{d}$ represening the banana orbit width of trapped particles and drift orbit width of passing particles. Here, $k_{\perp}$ and the banana orbit width of trapped particles are measured as in Fig. 10, while the drift orbit width of passing particles is measured for resonant test particles, including the correction due to finite Shafranov shift.

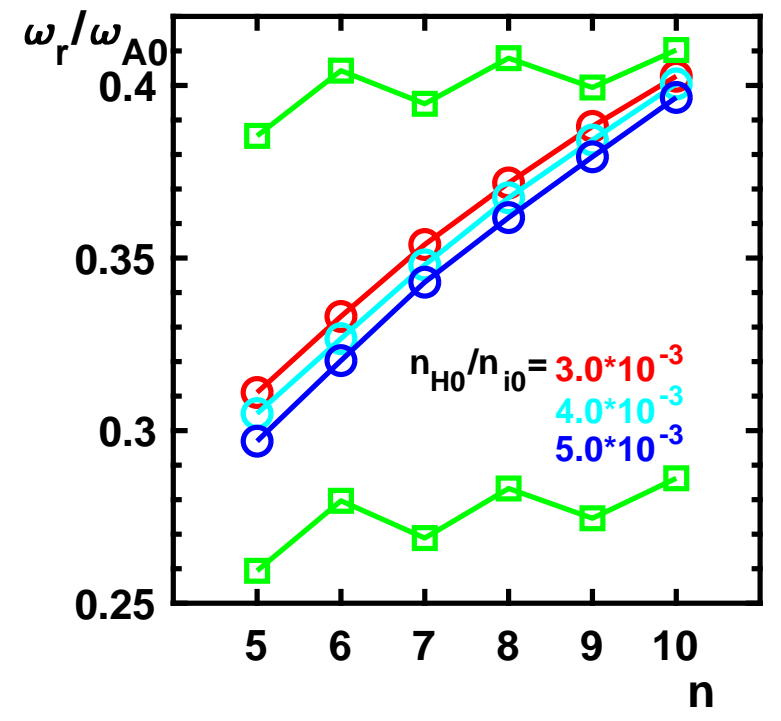

FIG. 19. Real frequencies $\omega_{r}$ of the TAE fluctuations for the same cases considered in Fig. 16. The respective upper and lower TAE accumulation points are shown in green curves.

Thus, we may expect a predominant role of transit resonances with passing EPs in the outer core region, with the most unstable mode number being $n \gtrsim O(10)$.

Real frequencies of the TAE fluctuations are shown in Fig. 19, for the same cases considered in Fig. 16, along with the respective upper and lower TAE accumu- lation points based on the radial locations of the fluctuations. The mode frequency increases with increasing $n$, and it goes closer to the upper SAW continuum. In the high mode number limit, the EP effect becomes non-perturbative even for the weakest drive case, as the separation of mode frequency and upper accumulation point becomes very small. The stronger damping associated with stronger coupling to SAW continuum, then provides another factor to be considered for the most unstable modes, in addition to the $k_{\perp} \rho_{d} \sim O(1)$ analysis discussed in great detail. Furthermore, we may speculate that the upper branch of kinetic TAEs ${ }^{50}$ instead of TAE could be driven unstable for even higher mode number.

\section{SUMMARY AND DISCUSSION}

In this paper, linear stability properties and shear Alfvén fluctuation spectra of a typical DTT reference scenario have been investigated by numerical simulations using hybrid MHD-gyrokinetic code HMGC, assuming a FAST-reference equilibrium and ITER-like EP parameters. In our simulations, a relatively simple geometry is assumed, and a simplified version of the simulation model of core plasmas is also adopted. This, of course, is far from being complete. For example, the analysis in this paper could be extended to realistic geometry and to include the kinetic effects of thermal plasmas, such as the compressibility and diamagnetic effects of thermal ions, which are able to play an important role in low frequency dynamics. These physics will be addressed in future analyses. Meanwhile, the present study retains the essential elements for analyzing the general features of Alfvénic fluctuation spectra resonantly excited by EPs in DTT. In particular, the peculiar meso-scale features due to the typical dimensionless EP orbit widths, similar to those expected in burning fusion plasmas; and generally smaller than in present day tokamaks.

We have shown that, due to the radial profile of adopted equilibrium and the resulting SAW continuum structure, the linear dynamics of the investigated scenario can be schematically divided into a central core region with nearly flat $q$ profile, and an outer core region with finite magnetic shear. The Alfvénic fluctuations in the central core region are shown to be dominated by low frequency, nearly undamped RSAEs, which are predominately driven unstable by the precession resonance of magnetically trapped particles, as identified by test particle analysis. Meanwhile, radiative damping is an important mechanism in the wave dissipation. The non-perturbative effect of EPs is shown to be evident for the RSAEs in lower $n$ cases, as $\Delta \omega_{\mathrm{EP}} \gtrsim \Delta \omega_{\mathrm{SAW}}$, i.e., the EP induced frequency shift is comparable or higher than the frequency mismatch of the eigenmode frequency with respect to the SAW accumulation point frequency. In the higher $n$ cases, the non-perturbative response is less evident, since the modes can be separate from SAW continuum. The most unstable toroidal mode number is 
$\sim O(10)$, consistent with the theoretical estimate based on the dimensionless EP orbit width, i.e., $k_{\perp} \rho_{d} \sim 1$. The wave particle power transfer is relatively broad and limited by the finite radial mode width rather than by the precession resonance condition of RSAE with magnetically trapped EPs. This, together with high toroidal mode numbers and the non-perturbative EP response, suggests that thermal plasma profiles should be corrugated on these length scales over characteristic times that are consistent with EP transport. As anticipated in the Introduction, this suggests the important role played by meso-scale physics in EP transport and nonlinear dynamics, which will be addressed in a separate work.

In the outer core region, higher frequency TAE modes are excited, for which the dominant dissipation is continuum damping due to the finite coupling with the SAW continuous spectrum. In the presence of the relative stronger damping than in the central core region, higher $n(n \geq 5)$ TAEs with stronger EP drive can be driven unstable for the reference EP profile with $n_{H 0} / n_{i 0}=3.0 \times 10^{-3}$. The effect of EPs driving TAE fluctuations is perturbative in lower $n$ cases, and becomes non-perturbative for higher- $n$ 's with the mode frequency being very close to the upper accumulation point frequency. Both precession resonance of trapped particles and transit harmonic resonances of passing particles contribute comparably to the overall mode drive. However, the features of wave-EP power transfer are significantly different for the two types of particles. This can be explained by their different resonance frequency radial profiles. The most unstable toroidal mode number for TAE fluctuations is $n \gtrsim 10$, even higher than RSAE fluctuations in the central core region and much larger than that of present day tokamaks. This is consistent with maximization of the combined contribution from trapped and passing particles, estimated by the $k_{\perp} \rho_{d} \sim 1$ criterion. Note that the characteristic normalized EP orbit widths in DTT, namely, banana orbit width of trapped particles and the drift orbit width of passing particles, are expected to be smaller than in present day tokamaks. Therefore, the most unstable mode numbers of SAW fluctuations in our simulations are consistent with DTT target design. This, again, suggests the important role played by meso-scale physics in EP transport and nonlinear dynamics, which, however, will be different for magnetically trapped and passing EPs. These issues are of great importance but are outside the intended scope of the present work, and will be subject of future investigations.

In the perspective of further research, crucial elements in the assessment of DTT are connected with nonlinear behaviors of Alfvénic fluctuations, such as the nonlinear saturation mechanism, fluctuation induced particle transport and global EP confinement. Notwithstanding the intrinsic limitations of the linear study presented in this work, our numerical simulation results are an important and necessary step towards a full nonlinear investigation. In fact, they clearly point out which nonlinear physics could be expected, and could play an important role for the operation of DTT. In particular, this analysis indicates the importance of meso-scales in nonlinear transport processes, and the unique role of EPs as mediators of cross-scale couplings. As a matter of fact, meso-scales naturally emerge due to the high toroidal mode number of the resonantly excited fluctuations spectrum. These are the length scales on which, based on the present theoretical understanding of nonlinear physics of EP and Alfvén waves, zonal structures will be excited and regulate drift wave turbulence and turbulent transport with a feedback on the fusion reactivity profiles. Meanwhile, the spatial structures of wave-EP power transfer will be reflected by the fluctuation induced EP transport. Thus, it is legitimate to expect that magnetically trapped $\mathrm{EP}$ give rise to more coherent nonlinear behaviors and, possibly, secular transport events; while passing particles should be very likely dominated by diffusive relaxation of radial profiles connected with the local fluctuation intensity. All these physics will be important elements to investigate in the future for understanding the physics in DTT. Nonetheless, this work can claim having illustrated the richness of the substantial physics which could be studied in the DTT device, as DTT is expected to be able to study integrated physics and technology aspects in burning plasma relevant conditions.

\section{ACKNOWLEDGMENTS}

One of the authors (T. Wang) is indebted to Professor Z. W. Ma for long-time continued support and many helpful discussions. The authors would like to thank C. Di Troia for in depth discussions of aspects of gyrokinetic simulations of Alfvénic fluctuations in burning plasmas. Part of this work was done during T. Wang's visit to ENEA Frascati Research Center. This work was carried out within the framework of the EUROfusion Consortium and received funding from the EURATOM research and training programme 2015-2018 under Grant Agreement No. 633053 (projects WP15-ER/ENEA-03 and WP17-ER/MPG-01). The views and opinions expressed herein do not necessarily reect those of the European Commission. This work was also supported by ITER-CN projects under Grants Nos. 2013GB104004 and 2013GB111004. The computing resources and the related technical support used for this work have been provided by CRESCO/ENEAGRID High Performance Computing infrastructure and its staff ${ }^{53}$.

\footnotetext{
${ }^{1}$ A. Pizzuto, ed., DTT: Divertor Tokamak Test facility - Project Proposal (ENEA Frascati Research Center, Via Enrico Fermi 45, 00044 Frascati (Roma), Italy, 2015).

${ }^{2}$ R. Albanese, A. Pizzuto, WPDTT2 Team, and DTT project proposal contributors, Fusion Eng. Des. 122, 274 (2017).

${ }^{3}$ R. Albanese and H. Reimerdes, Fusion Eng. Des. 122, 285 (2017).

${ }^{4}$ F. Crisanti, R. Albanese, R. Ambrosino, G. Calabro, B. Duval, G. Giruzzi, G. Granucci, G. Maddaluno, G. Ramogida, H. Reimerdes, and R. Zagorski, Fusion Eng. Des. 122, 288
} (2017). 
${ }^{5}$ I. Chapman, R. Kemp, and D. Ward, Fusion Eng. Des. 86, 141 (2011).

${ }^{6} \mathrm{~K}$. Tomabechi, J. Gilleland, Y. Sokolov, R. Toschi, and ITER Team, Nucl. Fusion 31, 1135 (1991).

${ }^{7}$ R. Aymar, V. Chuyanov, M. Huguet, R. Parker, Y. Shimamura, and the ITER Joint Central Team and Home Teams, in Proceedings of the 16th International Conference on Fusion Energy 1996, Vol. 1 (International Atomic Energy Agency, Vienna, 1997) p. 3.

${ }^{8}$ A. Pizzuto, F. Gnesotto, M. Lontano, R. Albanese, G. Ambrosino, M. Apicella, M. Baruzzo, A. Bruschi, G. Calabrò, A. Cardinali, R. Cesario, F. Crisanti, V. Cocilovo, A. Coletti, R. Coletti, P. Costa, S. Briguglio, P. Frosi, F. Crescenzi, V. Coccorese, A. Cucchiaro, C. Di Troia, B. Esposito, G. Fogaccia, E. Giovannozzi, G. Granucci, G. Maddaluno, R. Maggiora, M. Marinucci, D. Marocco, P. Martin, G. Mazzitelli, F. Mirizzi, S. Nowak, R. Paccagnella, L. Panaccione, G. Ravera, F. Orsitto, V. P. Ridolfini, G. Ramogida, C. Rita, M. Santinelli, M. Schneider, A. Tuccillo, R. Zagórski, M. Valisa, R. Villari, G. Vlad, and F. Zonca, Nucl. Fusion 50, 095005 (2010).

${ }^{9}$ L. Chen and F. Zonca, Rev. Mod. Phys. 88, 015008 (2016).

${ }^{10}$ Y. Song, S. Wu, J. Li, B. Wan, Y. Wan, P. Fu, M. Ye, J. Zheng, K. Lu, X. Gao, S. Liu, X. Liu, M. Lei, X. Peng, and Y. Chen, IEEE Trans. Plasma Sci. 42, 503 (2014).

${ }^{11}$ B. Wan, S. Ding, J. Qian, G. Li, B. Xiao, and G. Xu, IEEE Trans. Plasma Sci. 42, 495 (2014).

${ }^{12}$ S. Sharapov, B. Alper, H. Berk, D. Borba, B. Breizman, C. Challis, I. Classen, E. Edlund, J. Eriksson, A. Fasoli, E. Fredrickson, G. Fu, M. Garcia-Munoz, T. Gassner, K. Ghantous, V. Goloborodko, N. Gorelenkov, M. Gryaznevich, S. Hacquin, W. Heidbrink, C. Hellesen, V. Kiptily, G. Kramer, P. Lauber, M. Lilley, M. Lisak, F. Nabais, R. Nazikian, R. Nyqvist, M. Osakabe, C. P. von Thun, S. Pinches, M. Podesta, M. Porkolab, K. Shinohara, K. Schoepf, Y. Todo, K. Toi, M. Van Zeeland, I. Voitsekhovich, R. White, V. Yavorskij, ITPA EP TG, and JET-EFDA Contributors, Nucl. Fusion 53, 104022 (2013).

${ }^{13}$ N. Gorelenkov, S. Pinches, and K. Toi, Nucl. Fusion 54, 125001 (2014).

${ }^{14}$ P. Lauber, Phys. Rep. 533, 33 (2013).

${ }^{15}$ A. Fasoli, S. Brunner, W. A. Cooper, J. P. Graves, P. Ricci, O. Sauter, and L. Villard, Nat. Phys. 12, 411 (2016)

${ }^{16}$ C. Z. Cheng, L. Chen, and M. S. Chance, Ann. Phys. (N.Y.) 161, 21 (1985).

${ }^{17}$ L. Chen, Phys. Plasmas 1, 1519 (1994).

${ }^{18}$ F. Zonca and L. Chen, Phys. Plasmas 21, 072120 (2014).

${ }^{19}$ F. Zonca and L. Chen, Phys. Plasmas 21, 072121 (2014).

${ }^{20} \mathrm{~S}$. Briguglio, G. Vlad, F. Zonca, and C. Kar, Phys. Plasmas 2, 3711 (1995).

${ }^{21}$ S. Briguglio, F. Zonca, and G. Vlad, Phys. Plasmas 5, 3287 (1998)

${ }^{22}$ S. Briguglio, X. Wang, F. Zonca, G. Vlad, G. Fogaccia, C. Di Troia, and V. Fusco, Phys. Plasmas 21, 112301 (2014).

${ }^{23}$ X. Wang, S. Briguglio, P. Lauber, V. Fusco, and F. Zonca, Phys. Plasmas 23, 012514 (2016).

${ }^{24}$ X. Wang and S. Briguglio, New J. Phys. 18, 085009 (2016).

${ }^{25}$ G. Vlad, V. Fusco, S. Briguglio, G. Fogaccia, F. Zonca, and X. Wang, New J. Phys. 18, 105004 (2016).

${ }^{26}$ S. Briguglio, M. Schneller, X. Wang, C. Di Troia, T. HaywardSchneider, V. Fusco, G. Vlad, and G. Fogaccia, Nucl. Fusion 57, 072001 (2017).

${ }^{27}$ G. Vlad, S. Briguglio, G. Fogaccia, V. Fusco, C. Di Troia, E. Giovannozzi, X. Wang, and F. Zonca, "Single-n versus multiple-n simulations of Alfvénic modes," Submitted to Nucl. Fusion.

${ }^{28}$ G. Vlad, S. Briguglio, G. Fogaccia, F. Zonca, and M. Schneider, Nucl. Fusion 46, 1 (2006).

${ }^{29}$ H. Ninomiya, A. Kitsunezaki, M. Shimizu, M. Kuriyama, JT-60 Team, H. Kimura, H. Kawashima, K. Tsuzuki, M. Sato, N. Isei, Y. Miura, K. Hoshino, K. Kamiya, T. Ogawa, H. Ogawa, K. Miyachi, JFT-2M Group, S. Itoh, N. Yoshida, K. Hanada, K. Nakamura, H. Zushi, M. Sakamoto, E. Jotaki, M. Hasegawa, and Triam Group, Fusion Sci. Technol. 42, 7 (2002).

${ }^{30}$ A. Kitsunezaki, M. Shimizu, H. Ninomiya, M. Kuriyama, and THE JT-60 TEAM, Fusion Sci. Technol. 42, 179 (2002).

${ }^{31}$ S. Briguglio, G. Fogaccia, G. Vlad, and F. Zonca, Phys. Plasmas 14, 055904 (2007).

${ }^{32}$ W. Heidbrink, M. Van Zeeland, M. Austin, K. Burrell, N. Gorelenkov, G. Kramer, Y. Luo, M. Makowski, G. McKee, C. Muscatello, R. Nazikian, E. Ruskov, W. Solomon, R. White, and Y. Zhu, Nucl. Fusion 48, 084001 (2008).

${ }^{33}$ G. Vlad, S. Briguglio, G. Fogaccia, F. Zonca, C. Di Troia, W. Heidbrink, M. Van Zeeland, A. Bierwage, and X. Wang, Nucl. Fusion 49, 075024 (2009).

${ }^{34}$ A. Cardinali, M. Baruzzo, C. Di Troia, M. Marinucci, A. Bierwage, G. Breyiannis, S. Briguglio, G. Calabrò, G. Fogaccia, P. Mantica, G. Vlad, X. Wang, F. Zonca, V. Basiuk, R. Bilato, M. Brambilla, F. Imbeaux, S. Podda, and M. Schneider, in Fusion Energy (2010) Proc. 23rd IAEA Fusion Energy Conf., THW/P7-04 (International Atomic Energy Agency, Vienna, 2010).

${ }^{35}$ C. Di Troia, S. Briguglio, G. Fogaccia, G. Vlad, and F. Zonca, in Fusion Energy Conference 2012, Proc. 24th IAEA Fusion Energy Conf., TH/P6-21 (International Atomic Energy Agency, San Diego, 2012).

${ }^{36}$ F. Zonca, L. Chen, S. Briguglio, G. Fogaccia, A. V. Milovanov, Z. Qiu, G. Vlad, and X. Wang, Plasma Phys. Control. Fusion 57, 014024 (2015).

${ }^{37}$ L. Chen, R. B. White, and M. N. Rosenbluth, Phys. Rev. Lett. 52, 1122 (1984).

${ }^{38}$ R. Izzo, D. A. Monticello, W. Park, J. Manickam, H. R. Strauss, R. Grimm, and K. McGuire, Phys. Fluids 26, 2240 (1983).

${ }^{39}$ X. Wang, S. Briguglio, L. Chen, G. Fogaccia, G. Vlad, and F. Zonca, Phys. Plasmas 18, 052504 (2011).

${ }^{40}$ W. Park, S. Parker, H. Biglari, M. Chance, L. Chen, C. Z. Cheng, T. S. Hahm, W. W. Lee, R. Kulsrud, D. Monticello, L. Sugiyama, and R. White, Phys. Fluids B 4, 2033 (1992).

${ }^{41}$ T. H. Stix, Plasma Phys. 14, 367 (1972).

${ }^{42}$ X. Wang, F. Zonca, and L. Chen, Plasma Phys. Control. Fusion 52, 115005 (2010).

${ }^{43}$ W. Deng, Z. Lin, I. Holod, X. Wang, Y. Xiao, and W. Zhang, Phys. Plasmas 17, 112504 (2010).

${ }^{44}$ F. Zonca, L. Chen, S. Briguglio, G. Fogaccia, G. Vlad, and X. Wang, New J. Phys. 17, 013052 (2015).

${ }^{45}$ R. R. Mett and S. M. Mahajan, Phys. Fluids B 4, 2885 (1992).

${ }^{46}$ R. White, Commun. Nonlinear Sci. Numer. Simulat. 17, 2200 (2012).

${ }^{47}$ G. Y. Fu and C. Z. Cheng, Phys. Fluids B 4, 3722 (1992).

${ }^{48}$ H. L. Berk, B. N. Breizman, and H. Ye, Phys. Lett. A 162, 475 (1992).

${ }^{49}$ S. T. Tsai and L. Chen, Phys. Fluids B 5, 3824 (1993).

${ }^{50}$ F. Zonca and L. Chen, Phys. Plasmas 3, 323 (1996).

${ }^{51}$ F. Zonca and L. Chen, Phys. Rev. Lett. 68, 592 (1992).

${ }^{52}$ F. Zonca and L. Chen, Phys. Fluids B 5, 3688 (1993).

${ }^{53} \mathrm{G}$. Ponti et al, in the 2014 International Conference on High Performance Computing and Simulation, HPCS 2014, art. no. 6903807, 1030-1033 (2014). 